\title{
Art. 91c GG als archimedischer Punkt staatlicher Informationsverarbeitung und Wissensgenerierung im Bundesstaat
}

von Utz Schliesky

\section{Einführung}

Die Digitalisierung von Staat und Gesellschaft schreitet unaufhaltsam voran. Die moderne Informations- und Kommunikationstechnik verändert nicht nur in atemberaubender Geschwindigkeit gesellschaftliche Kommunikationsbeziehungen sowie soziale und ,realwirtschaftliche“ Strukturen, sondern auch und gerade die Rolle des Staates. ${ }^{1}$ Dessen Erscheinungsbild ist dabei vielschichtig: Negativbeispiele wie die geheimdienstliche Ausforschung sämtlicher InternetKommunikation $^{2}$ zeichnen das Bild von einem freiheitsbedrohenden Leviathan, während die Daten-Sammelwut privater Internet-Konzerne den fürsorglichen und Freiheitsschutz verbürgenden Staat auf den Plan ruft. Schließlich und vor allem finden sich zahlreiche alltägliche Bemühungen im Bereich des $E$ Government, also der Binnenmodernisierung von Staat und Verwaltung mithilfe der Informations- und Kommunikationstechnik. Trotz dieser und zahlreicher weiterer Facetten staatlichen Tätigwerdens in der virtuellen Welt ist aber oftmals zu konstatieren, dass staatliche Einheiten den Entwicklungen in Wirtschaft und Gesellschaft deutlich hinterherlaufen. Gerade mit Blick auf elektronische Geschäftsprozesse wird die Kluft zwischen Staat einerseits und Gesellschaft sowie Wirtschaft andererseits eher größer als kleiner. Die Rechtsordnung und gerade auch die Verfassung haben hier erheblichen Nachholbedarf, wenn die Steuerungsfunktion des Rechts erhalten bleiben soll. ${ }^{3}$ Dieser Nachholbedarf kann auch

1 Zu den Auswirkungen auch Schulz, S.E.: Die „Datenautobahn“ als Infrastruktur: Gewährleistungs- und Verkehrssicherungspflichten des Staates, in: Hill, H./Schliesky, U. (Hg.): Die Vermessung des virtuellen Raumes, 1. Aufl., Baden-Baden, 2012, 265-305, hier 270 ff.

2 Man denke an das amerikanische PRISM-Programm oder das britische TEMPORA-Projekt.

3 Zur Rolle des Rechts bei staatlichen Innovationen Richter, E.: Die Bedeutung des Rechts im staatlichen Innovationsmanagement - am Beispiel der Umsetzung von E-Government, in: Schliesky, U. (Hg.): Staatliches Innovationsmanagement, Kiel, 2010, 157-183. 
nicht überraschen, geht es doch um neue Organisations- und Verfahrensmöglichkeiten, die sich von dem an einer physischen Präsenz und dem Prinzip der Schriftlichkeit orientierten Bauprinzip der Verwaltung deutlich unterscheiden.

Als Ergebnis der Föderalismuskommission II wurde im Jahr 2009 die Informations- und Kommunikationstechnik in Gestalt des Art. 91c GG erstmals in das Grundgesetz aufgenommen. ${ }^{4}$ So bedeutsam und zugleich begrüßenswert dieser Schritt war, so sehr trägt die Vorschrift doch Kompromisszüge ${ }^{5}$ und regelt zudem nur bestimmte Aspekte der IT-Kooperation zwischen Bund und Ländern. Angesichts des „Heraufziehens“ einer Föderalismusreform III, die schon aus finanzverfassungsrechtlichen Gründen unabdingbar ist, erscheint es an der Zeit, eine erste Bilanz des Art. 91c GG zu ziehen sowie einen vorsichtigen Blick auf weitere Reformbedarfe zu werfen.

\section{Bilanz: vier Jahre Art. 91c GG}

\section{Bedeutung der Norm}

Mit Art. 91c GG hat die Informations- und Kommunikationstechnik erstmals Eingang in das Grundgesetz gefunden. Die Aufnahme dieser Vorschrift ist ein wichtiger Schritt zur Angleichung der Verfassungsrechtslage an gewandelte technische Möglichkeiten und gesellschaftliche Rahmenbedingungen. Der Anwendungsbereich ist allerdings begrenzt; Art. 91c GG betrifft nur die IT-Systeme von Bund und Ländern. Die Länder ihrerseits sind aber in der Pflicht, sekundäroder tertiärrechtlich auf Art. 91c GG beruhende Regelungen, wie z.B. vereinbarte technische Standards gegenüber ihren Kommunen und anderen Trägern der öffentlichen Verwaltung, verbindlich umzusetzen. ${ }^{6}$ Die gesellschaftliche Sphäre ${ }^{7}$ harrt hinsichtlich der IT auch weiterhin der verfassungsrechtlichen Prägung, die

4 Art. 91c GG war als Ergebnis der Föderalismuskommission II Bestandteil des 57. Gesetzes zur Änderung des Grundgesetzes vom 29. 07. 2009 (BGB1. I, 2248) und ist am 01. 08. 2009 in Kraft getreten.

5 Schliesky,U.: in: Hofmann, H./Hopfhauf, A. (Hg.): Bonner Kommentar zum Grundgesetz, 12. Aufl., Köln, 2011, Art. 91c Rn. 13.

6 Zur Umsetzungsmöglichkeit durch E-Government-Gesetze: Schulz, S.E.: Macht Art. 91c Grundgesetz EGovernment-Gesetze der Länder erforderlich?, in: Die Öffentliche Verwaltung, 10/61 (2010), 225-229; zur Rechtsnatur der Beschlüsse des IT-Planungsrates Schulz, S.E./Tallich, M.: Rechtsnatur des ITStaatsvertrages und seiner Beschlüsse, in: Neue Zeitschrift für Verwaltungsrecht, 21/29 (2010), 13381342.

7 Zur Digitalisierung des Alltags Richter, I.: Die Digitalisierung des Alltags, in: Mehde, V./Ramsauer, U./Seckelmann, M. (Hg.): Staat, Verwaltung, Information, Festschrift für Hans Peter Bull zum 75. Geburtstag, Berlin, 2011, 1041-1056. 
derzeit allein der Rechtsfortbildung durch das Bundesverfassungsgericht ${ }^{8}$ und anderer Gerichte (vor allem dem BGH) überlassen ist. ${ }^{9}$

Die bisherige IT-Steuerung im föderalen Staat Bundesrepublik Deutschland hat sich zunehmend als problematisch erwiesen und wurde im Hinblick auf Effektivität, Wirtschaftlichkeit, Reaktionsgeschwindigkeit und den Vergleich mit internationalen Standards als unzureichend empfunden. ${ }^{10}$ Im Laufe der Jahre ist eine heterogene, nicht einmal im Ansatz gemeinsam geplante, abgestimmte und dementsprechend auch nicht kompatible (,,interoperable“) IT-Infrastruktur der öffentlichen Verwaltung in Deutschland entstanden. ${ }^{11}$ Vergleichbares gilt für die ITAnwendungen, da jeder Aufgabenträger aufgrund seiner umfassenden Aufgabenerledigungskompetenz eigenständig über das $\mathrm{Ob}$ und Wie von IT-Anwendungen und damit auch die Beschaffung verschiedenster Software-Produkte entscheidet. Mittlerweile hat jede Gebietskörperschaft mehr oder weniger ITLösungen bei der Aufgabenerfüllung im Einsatz. Neben erheblichen, häufig unnötigen Ausgaben ${ }^{12}$ ist die Bundesrepublik Deutschland auch kaum noch in der Lage, europarechtliche Vorgaben im Bereich des E-Government umzusetzen. Ein aktuelles Beispiel bietet Art. 8 EU-Dienstleistungsrichtlinie, der für einen relevanten Bereich der öffentlichen Verwaltung eine elektronische Abwicklung erfordert, ${ }^{13}$ die innerstaatlich noch nicht geleistet wurde. ${ }^{14}$ Die Schaffung einer

8 Allen voran die Neuschöpfung des „Grundrechts auf Gewährleistung der Vertraulichkeit und Integrität informationstechnischer Systeme“ in der Entscheidung zur „Online-Durchsuchung“, BVerfGE 120, 274 ff.; dazu Britz, G.: Vertraulichkeit und Integrität informationstechnischer Systeme, in: Die Öffentliche Verwaltung, 10/61 (2008), S. 411-415; Heckmann, D.: Der virtuelle Raum als Wohnung?, in: Kluth, W./Müller, M./Peilert, A. (Hg.): Wirtschaft - Verwaltung - Recht, Festschrift für Rolf Stober, Köln u. a., 2008, 615-632; Hoffmann-Riem, W.: Der grundrechtliche Schutz der Vertraulichkeit und Integrität eigener informationstechnischer Systeme, in: Juristenzeitung, 21/63 (2008), 1009-1022. S. ferner BVerfGE 121, 1 ff. („Automatisierte KFZ-Kennzeichen Erkennung“); 123, 39 ff. („elektronische Wahlgeräte"); 125, 260 ff. (,Vorratsdatenspeicherung“).

9 S. etwa BGH, NJW 2012, 148 ff. (Löschungsanspruch bei Persönlichkeitsrechtsverletzung in Blogs); BGH, Urteil vom 24. 01. 2013 - III ZR 98/12 = NJW 2013, 1072 ff. (Internet als Grundversorgung); BGH, Urteil vom 14. 05. 2013, Az. VI ZR 269/12 = VersR 2013, 771 ff. (Autocomplete-Funktion und Persönlichkeitsrechte).

10 Ruge, K., in: Schmidt-Bleibtreu, B./Hofmann, H./Hopfauf, A. (Hg.): Grundgesetz, 12. Aufl., Köln, 2011, Art. 91c Rn. 2; Schallbruch, M./Städler, M.: Neuregelung der Bund-Länder-Zusammenarbeit bei der IT durch Art. 91c GG, in: Computer und Recht, 9/25 (2009), 619-624, hier 619 f.; Schliesky, U.: Verfassungsrechtliche Rahmenbedingungen des E-Government, in: Die Öffentliche Verwaltung, 19/57 (2004), 809-818, hier $812 \mathrm{ff}$.

11 Zutreffend Schallbruch, M./Städler, M.: a. a. O., 620.

12 Insgesamt werden für die öffentliche IT geschätzt $15 \mathrm{Mrd}$. Euro/Jahr ausgegeben, s. Schallbruch, M.Städler, M.: a. a. O., 619.

$13 \mathrm{Zu}$ Inhalt und Umfang der Verpflichtung Luch, A.D./Schulz, S.E.: Vorgaben der EUDienstleistungsrichtlinie zur elektronischen Verfahrensabwicklung, in: Schliesky, U. (Hg.): Die Umset- 
flächendeckenden, interoperablen Vernetzung der öffentlichen Verwaltung in Deutschland und damit eines ebenenübergreifenden E-Government stellen eine gravierende Herausforderung für die föderale Eigenstaatlichkeit von Bund und Ländern dar, der schon aus technischen Gründen - noch mehr als bei der herkömmlichen, an der Schriftform orientierten Verwaltung - nur mit viel stärkerer Abstimmung und Koordinierung begegnet werden kann. Dieser Bedarf an engerer Abstimmung zwischen Bund und Ländern sowie verbindlicher Koordinierung läuft naturgemäß den Entflechtungsbestrebungen ${ }^{15}$ der Föderalismusreformen I und II zuwider. ${ }^{16}$ Grundlage des Art. 91c GG ist daher die Erkenntnis, dass erhebliche Verbesserungsbedarfe bei der IT-Steuerung, vor allem bezüglich Infrastrukturen, Sicherheit und Interoperabilität, sowie der zunehmende Wunsch von Gebietskörperschaften nach gemeinschaftlicher Aufgabenerledigung bzw. dem gemeinsamen Betrieb von IT-Anwendungen bestehen. ${ }^{17}$

Die Norm des Art. 91c GG ist nunmehr die zentrale Grundlage des föderalen $E$ Government, die aus Sicht der meisten IT- und E-Government-Verantwortlichen überfällig war. Dabei darf nicht verschwiegen werden, dass die Vorschrift angesichts der sehr unterschiedlichen Vorstellungen der relevanten Akteure in der Föderalismusreformkommission II Kompromisscharakter aufweist. Die prägende Kraft der Norm ist daher nicht zu überschätzen; nach wie vor entziehen sich Ressortegoismen und „Fachbruderschaften“ der verfassungsrechtlich gewollten Koordinierung, wie die Schaffung des „E-Justice-Rats“ in Konkurrenz zum IT-

zung der EU-Dienstleistungsrichtlinie in der deutschen Verwaltung, Teil II: Verfahren, Prozesse, ITUmsetzung, Kiel, 2009, 219-321, hier 272 ff.; Schulz, S.E.: Gemeinschaftsrechtliche Verpflichtung zur elektronischen Verfahrensabwicklung (Art. 8 DLR) als Perspektive zur Etablierung eines Rechtsrahmens des eGovernment?, in: Deutsches Verwaltungsblatt, 1/124 (2009), 12-21. Schliesky, U.: Von der Realisierung des Binnenmarkts über die Verwaltungsreform zu einem Gemeineuropäischen Verwaltungsrecht? Die Auswirkungen der geplanten EU-Dienstleistungsrichtlinie auf das deutsche Verwaltungsrecht, in: Deutsches Verwaltungsblatt, 14/120 (2005), 887-895, hier 890; ders.: Die Europäisierung der Amtshilfe, Stuttgart u. a., 2008, 41 ff.; Ziekow, J./Windoffer, A., in: Schlachter, M./Ohler, C. (Hg.): Europäische Dienstleistungsrichtlinie, 1. Aufl., Baden-Baden, 2008, Art. 8 Rn. 2 ff.

14 Die EU-Kommission hatte ein Vertragsverletzungsverfahren u. a. gegen die Bundesrepublik Deutschland eingeleitet, s. die Pressemitteilung der Europäischen Kommission vom 27. 10. 2011, Ref.: IP/11/1283. Die Mitteilung der formellen Umsetzungsmaßnahmen hat die EU-Kommission veranlasst, dieses Verfahren am 26. 01. 2012 einzustellen.

$15 \mathrm{Zu}$ diesen Huber, P.M.: Klarere Verantwortungsteilung von Bund, Ländern und Kommunen?: Gutachten D zum 65. Deutschen Juristentag, München, 2004, 11 ff., $33 \mathrm{ff}$.

16 Zutreffend Ruge, K., a. a. O., Art. 91c Rn. 1; Seckelmann, M.: „Renaissance“ der Gemeinschaftsaufgaben in der Föderalismusreform II?, in: Die Öffentliche Verwaltung, 18/62 (2009), 747-757, hier 753.

17 Dazu Heckmann, D.: Grundgesetz 2.0: Staat und IT in guter Verfassung?, in: Kommunikation \& Recht, 1/12 (2009), 1-7, hier 2; Schallbruch, M./Städler, M., a. a. O., 620, 624; Schliesky, U.: Verfassungsrechtliche Rahmenbedingungen, a. a. O., 812 ff. 
Planungsrat zeigt. ${ }^{18}$ Wenigstens aber beseitigt der Kooperationstatbestand des Art. 91c Abs. $1 \mathrm{GG}$ das bislang bestehende verfassungsrechtliche Hindernis für eine engere Zusammenarbeit zwischen Bund und Ländern, einschließlich gemeinsamer Beschaffungen. ${ }^{19}$

Über den nur deklaratorischen oder gerade konstitutiven Charakter von Art. 91c GG sowie über die Einordnung als Gemeinschaftsaufgabe herrschte schon während der Beratungen in der Föderalismuskommission II Uneinigkeit; diese setzt sich nun in der Kommentarliteratur fort. So wird in Art. 91c GG zum Teil kein unmittelbarer Verfassungsauftrag zur (stärkeren) IT-Zusammenarbeit der staatlichen Gebietskörperschaften, sondern lediglich eine ,fakultative Gemeinschaftsaufgabe“ gesehen. ${ }^{20}$ Eine derartige Qualifizierung greift jedoch zu kurz, enthält doch allein Art. 91c Abs. 4 GG eine ausschließliche Gesetzgebungskompetenz des Bundes nebst Zuweisung einer in bundeseigener Verwaltung zu erfüllenden Aufgabe. Darüber hinaus stellt sich bei Art. 91c GG das sehr grundlegende Problem, ob es sich bei IT um eine Aufgabe oder eine Funktion handelt. Letztlich entscheidet die Einordnung im Einzelfall über die (verfassungs)rechtlichen Anforderungen und zugleich praktisch über den Erfolg des Tätigwerdens der Verwaltung. Informations- und Kommunikationstechnik ist in der Regel gerade keine eigenständige Verwaltungsaufgabe, sondern eine Querschnittsfunktion der Verwaltung. ${ }^{21}$ Soweit es um Errichtung und Betrieb eines Verbindungsnetzes geht (Art. 91c Abs. 4 GG), fehlt es zudem am fakultativen Charakter. Formal wird durch Art. 91c Abs. 4 GG eine eindeutige Bundeskompetenz für Gesetzgebung und Verwaltung begründet, ${ }^{22}$ doch der Charakter der Aufgabe (Verbindung der Netzinfrastrukturen von Bund und Ländern) und die Konkretisierung im IT-Netz-Gesetz sowie die Abstimmung im IT-Planungsrat ${ }^{23}$ lassen Errichtung und Betrieb des Verbindungsnetzes gem. Art. 91c Abs. 4 GG zu einer faktischen Gemeinschaftsaufgabe werden. Richtigerweise ist bei der Einordnung von Art. 91c GG daher zu differenzieren: In vielen Bereichen weist Art. 91c wegen

18 Die entsprechende Vereinbarung über die Errichtung des aus den Justizstaatssekretären des Bundes und der Länder bestehenden E-Justice-Rats ist allerdings noch nicht ratifiziert worden.

19 Dazu Siegel, T.: Neue Querschnittsaufgaben und Gewaltenteilung, in: Der Staat, 2/49 (2010), 299-322, hier 321.

20 So Heun, W.: in: Dreier, H (Hg.): Grundgesetz, 2. Aufl., Supplementum 2010, Tübingen, 2010, Art. 91c Rn. 7; Seckelmann, M.: ,Renaissance“ der Gemeinschaftsaufgaben, a. a. O., 754; s. auch Siekmann, H.: in: Sachs, M. (Hg.): Grundgesetz, 6. Aufl., München, 2011, Art. 91c Rn. 6.

21 Insoweit inkonsequent, aber zutreffend Heun, W., a. a. O., Art. 91c Rn. 6.

22 Deutlich auch Ruge, K., a. a. O., Art. 91c Rn. 1 a. E.

23 S. § 4 IT-Staatsvertrag. 
seines Kompromisscharakters eine nur deklaratorische Bestätigung bereits zuvor erlaubter Kooperationen auf (insbesondere Abs. 1). ${ }^{24}$ Die Vereinbarung von Standards hingegen, die auf der Grundlage des IT-Staatsvertrags sogar mit qualifizierter Mehrheit für Bund und alle Länder verbindlich beschlossen werden können, sind ein Novum; hier weist die Norm konstitutive Wirkung auf. ${ }^{25}$ Und auch Art. 91c Abs. 3 GG beschreitet mit der Eröffnung einer Kooperationsmöglichkeit für Aufgabenteile verfassungsrechtliches Neuland. Art. 91c GG regelt streng genommen daher keine Gemeinschaftsaufgabe, sondern normiert eine Verantwortungsteilung für die Informations- und Kommunikationstechnik zwischen Bund und Ländern, ${ }^{26}$ die verfassungssystematisch den richtigen Standort in dem Abschnitt „Bund und die Länder“ gehabt hätte. ${ }^{27}$ Dem hat der Verfassungsgeber systematisch dadurch Rechnung getragen, dass er die Überschrift des Abschnitts VIIIa. um den Begriff „Verwaltungszusammenarbeit“ ergänzt hat. Bedeutung hat hier vor allem die gemeinsame Verantwortung von Bund und Ländern für die Festlegung von Interoperabilitäts- und Sicherheitsstandards, die vom IT-Planungsrat festzulegen sind.

\section{Praktische Umsetzung}

Art. 91c GG hat bereits einige nicht unerhebliche rechtliche und faktische Folgen ausgelöst. Zunächst einmal wurde von den verfassungsunmittelbaren Kompetenzen rasch Gebrauch gemacht. Bereits parallel zu der Grundgesetz-Änderung wurde das IT-Netz-Gesetz beraten und verabschiedet, ${ }^{28}$ das am 18. August 2009 in Kraft getreten ist. Der auf Art. 91c Abs. 2 GG beruhende IT-Staatsvertrag trat nach erfolgreichem Ratifizierungsverfahren am 1. April 2010 in Kraft. ${ }^{29}$ Mit dem IT-Staatsvertrag wurde auch der IT-Planungsrat eingerichtet, das Zusammenwir-

24 So wurde die verfassungsrechtliche Zulässigkeit der Staatssekretärsrunde Deutschland-Online vor Verabschiedung des Art. 91c GG nicht bezweifelt, obwohl dort Planung und Koordinierung von ITProjekten erfolgten und im Rahmen der Umsetzung der EU-Dienstleistungsrichtlinie sogar Einfluss auf die Ausgestaltung der $\S \S 71$ a ff. VwVfG genommen wurde.

25 Deutlich auch Hofmann, H/Schlief, L. (Hg.): Grundgesetz mit Begleitgesetz: die Föderalismusreform II mit neuen Gesetzestexten, Gesetzesbegründungen und einer Einführung, Heidelberg, 2009, XXVI.

26 Schallbruch, M./Städler, M., a. a. O., 621; allgemein zur Verantwortungsteilung Schliesky, U.: Souveränität und Legitimität von Herrschaftsgewalt, Tübingen, 2004, 534 ff.; im E-Government Schliesky, U.: Verantwortung im E-Government, in: ders./Schulz, S.E. (Hg.): Transparenz, Partizipation, Kollaboration - Web 2.0 für die öffentliche Verwaltung, Kiel, 2012, 1-10, hier $5 \mathrm{ff}$.

27 Zum Vorschlag von Art. 37a, 37b GG näher Schliesky, U.: Die Aufnahme der IT in das Grundgesetz, in: Zeitschrift für Staats- und Europawissenschaften, 6/6 (2008), 304-330, hier 325 ff.

28 BGB1. I 2009, 2702.

$29 \S 7$ Abs. 1 IT-Staatsvertrag; Zustimmungsgesetz des Bundes: BGB1. I 2010, 662. 
ken i. S. v. Art. 91c Abs. 1 GG erfolgt vor allem durch eine entsprechende Gremienstruktur. Bund und Länder haben mit Recht die Institutionalisierung der ITSteuerung für erforderlich gehalten. Dementsprechend erfolgt nun die gemeinsame Abstimmung zwischen Bund und Ländern in diesem neu errichteten Gremium. Es war bereits durch die Föderalismuskommission II im „Gemeinsamen Grundverständnis ${ }^{\text {‘30 }}$ vorgesehen und wurde im Rahmen der Kommissionsberatungen konzipiert.

Existenz und Tätigkeit des IT-Planungsrates auf einer verfassungsrechtlichen Basis bedeuten schon per se einen erheblichen Fortschritt gegenüber dem doch weitestgehend rechtsfreien Raum in den Jahren zuvor. Auch wenn das Vorläufergremium, die Staatssekretärsrunde E-Government ${ }^{31}$ durchaus gute Arbeit geleistet hat, so verfügt der IT-Planungsrat nun über ganz andere rechtliche Möglichkeiten. Er hat diese auch durchaus bereits genutzt und erste Erfolge, wie etwa die Verabschiedung der Nationalen E-Government-Strategie, verbuchen können. ${ }^{32}$ Das Zusammenwirken der Landes-IT-Verantwortlichen mit der Beauftragten der Bundesregierung für Informationstechnik erweist sich als segens- und erfolgreich, nicht zuletzt, weil Fach- und Ressortegoismen in diesem Gremium keinen Raum haben. Die Verabschiedung des E-Government-Gesetzes des Bundes, ${ }^{33}$ dem zweiten derartigen Gesetz nach der schleswig-holsteinischen Pionierregelung, ${ }^{34}$ unterstützt die Bemühungen des IT-Planungsrates zusätzlich. Dennoch bleibt die Wirksamkeit des Gremiums und seiner Beschlüsse durchaus noch verbesserungsfähig und -bedürftig. Dies sieht der IT-Planungsrat auch selbst so; er hat daher ein umfangreiches Gutachten zu den „Funktionen des ITPlanungsrats bei normgebenden Verfahren auf dem Gebiet von IT und $E$ -

30 Abgedruckt bei Schliesky, U.: Bonner Kommentar, a. a. O., Art. 91c, Anhang.

31 Dazu Schliesky, U.: Verwaltungsreform als Aufgabe, in: Verwaltungsarchiv, 3/98 (2008), 313-350, hier $321 \mathrm{f}$.

32 Beus, H.B.: Grundzüge für eine deutsche E-Government-Strategie, in: Wirtz, B.W. (Hg.): E-Government - Grundlagen, Instrumente, Strategien, Wiesbaden, 2010, 255-266; Wentzel, J.: Die Nationale EGovernment-Strategie: Ein Schritt vor, zwei zurück?, in: Verwaltung \& Management, 6/16 (2010), 283292.

33 Dazu Müller-Terpitz, R./Rauchhaus, A.: Das E-Government-Gesetz des Bundes - ein Schritt in Richtung „Verwaltung 2.0“, in: MultiMedia und Recht, 1/16 (2013), 10-16, hier 11 ff.; Habammer, C./Denkhaus, $W$.: Das E-Government-Gesetz des Bundes - Inhalt und erste Bewertung - Gelungener Rechtsrahmen für elektronische Verwaltung, in: MultiMedia und Recht, 6/16 (2013), 358-362.

34 GVOBl. SH 2009, 398; Schulz, S.E.: Ein eGovernment-Gesetz für Schleswig-Holstein - Angriff auf die kommunale Selbstverwaltung?, in: Die Gemeinde Schleswig-Holstein, 11/50 (2008), 272-278. 
Government" in Auftrag gegeben. ${ }^{35}$ Trotz erster erfolgreicher Maßnahmen muss der Rat weiter um seine Rollenfindung bemüht sein, da es vergleichbare Gremien mit einer derartigen Querschnittsfunktion nicht gibt. Insbesondere das Verhältnis zu Fachministerkonferenzen erweist sich nach wie vor als schwierig. Hinzu kommt, dass im Bereich der IT-Steuerung und Verwaltungsmodernisierung die maßgeblichen Akteure verhältnismäßig häufig wechseln und personelle Kontinuität nur bedingt gewährleistet ist. Auch sind die maßgeblichen Kompetenzen in den Ländern und im Bund jeweils unterschiedlich verortet sind, sodass die Aufgabenstellungen durchaus variieren. Zudem fehlt dem IT-Planungsrat bislang ein eigener Unterbau; die Geschäftsstelle ist zu geringfügig und zu oft wechselnd besetzt. Der fehlende Vorbereitungsapparat führt letztlich dazu, dass der Rat bislang kaum verbindliche Standardisierungen gem. Art. 91c Abs. 2 S. 1 GG verabschieden konnte. Und schließlich ist die Wahrnehmung der strategischen Zielsetzungsaufgabe durch den IT-Planungsrat noch unzureichend. Die erhoffte Rolle als Impulsgeber für organisations- und verfahrensrechtliche Veränderungen, die das E-Government ja ausmachen, wird bislang nicht ausgefüllt. Insoweit fehlt es an Impulsen für weitere rechtliche Veränderungen.

\section{IT in der öffentlichen Verwaltung und E-Government}

Die vom IT-Planungsrat festzulegenden Standards sind heutzutage unabdingbare Voraussetzung für die ebenenübergreifende Zusammenarbeit der Behörden von Bund, Ländern und Kommunen sowie im europäischen Behördennetz. ${ }^{36}$ Die Ausgangslage vor Schaffung des Art. 91c GG in Gestalt der heutigen Verwaltungsrealität war nicht mehr deckungsgleich und kaum noch in Einklang zu bringen mit dem im Grundgesetz erkennbaren Leitbild der öffentlichen Verwaltung. Das Grundgesetz geht schon ausweislich des Wortlauts von der ,vollziehenden Gewalt ${ }^{637}$ und damit von einer vor allem Gesetze ausführenden Verwaltung aus. Der Einsatz von IT und insbesondere der Umbau der Verwaltungsorganisation

35 Gutachten im Auftrag des IT-Planungsrats von Richter, E./Schmehl, A./Spiecker gen. Döhmann, I., abrufbar unter www.it-planungsrat.de.

36 Dazu Schliesky, U.: Von der organischen Verwaltung Lorenz von Steins zur Netzwerkverwaltung im Europäischen Verwaltungsverbund, in: Die Öffentliche Verwaltung, 16/62 (2009), 641-649, hier 647 f.; ders., Die Europäisierung der Amtshilfe, a. a. O., 13 ff.; Schmidt-Aßmann, E.: Einleitung: Der Europäische Verwaltungsverbund und die Rolle des Europäischen Verwaltungsrechts, in: ders./SchöndorfHaubold, B. (Hg.): Der Europäische Verwaltungsverbund, Tübingen, 2005, 1-24, hier 6 ff.; Sydow, G.: Verwaltungskooperation in der Europäischen Union, Tübingen, 2004, 78 ff.; Wettner, F.: Die Amtshilfe im Europäischen Verwaltungsrecht, Tübingen, 2005, 277 ff.

37 Art. 1 Abs. 3, Art. 20 Abs. 3, Art. 83 ff. GG. 
mithilfe von IT passt aber nur bedingt zu diesem Leitbild. Längst existiert - von verschiedenen Seiten (Europäische Union; Modernisierungsansätze von Bund, Ländern und Kommunen) - ein neues Leitbild der Verwaltung, das sehr stark auf elektronische Prozesse setzt und eben mit dem Begriff E-Government bzw. Electronic Government gekennzeichnet ist. ${ }^{38}$ Hinter diesem Begriff verbirgt sich der wohl grundlegendste Wandel der öffentlichen Verwaltung seit Jahrzehnten, womöglich seit Jahrhunderten. Denn mit dem Begriff des E-Government ist nicht lediglich die Eröffnung einer weiteren Zugangsmöglichkeit von Wirtschaft und Gesellschaft zur öffentlichen Verwaltung verbunden, sondern dank der Möglichkeiten der Informations- und Kommunikationstechnik eine ganz neue Perspektive zur Gestaltung von Verwaltungsverfahren, der Verwaltungsorganisation sowie der internen Arbeitsprozesse. ${ }^{39}$ Wie für andere Leitbegriffe der Verwaltungsmodernisierung gilt gerade auch für das E-Government, dass eine Vielzahl völlig unterschiedlicher Konzepte existiert, die bei Bund, Ländern und Kommunen noch längst nicht miteinander verzahnt sind. ${ }^{40}$ In Deutschland ist bislang die sog. Speyerer Definition vorherrschend, die E-Government als die „Abwicklung geschäftlicher Prozesse in Zusammenhang mit Regieren und Verwalten (Government) mithilfe von Informations- und Kommunikationstechniken

38 Ebenso Volkmann, U., in: von Mangoldt, H./Klein, F./Starck, C. (Hg.): Kommentar zum Grundgesetz, Band 3: Art. 83 bis 146, 6. Aufl., München, 2010, Art. 91c Rn. 1; Britz, G.: Elektronische Verwaltung, in: Hoffmann-Riem, W./Schmidt-Aßmann, E./Voßkuhle, A. (Hg.): Grundlagen des Verwaltungsrechts, Band II, 2. Aufl., München, 2012, § 26 Rn. 2 ff.; s. ferner Eifert, M.: Electronic Government, BadenBaden, 2006, $20 \mathrm{ff}$;; Groß, T.: Die Informationierung der Verwaltung, in: Verwaltungsarchiv, 3/95 (2004), 400-417; Schliesky, U.: Verwaltungsreform als Aufgabe, a. a. O., 339 f.; ders.: Auswirkungen des E-Government auf Verfahrensrecht und Kommunale Verwaltungsstrukturen, in: Neue Zeitschrift für Verwaltungsrecht, 11/22 (2003), 1322-1328.

39 Grundlegend Eifert, M., a. a. O., insbes. 33 ff.; Schliesky, U.: Auswirkungen des E.Government auf Verfahrensrecht und kommunale Verwaltungsstrukturen, in: Neue Zeitschrift für Verwaltungsrecht, 11/22 (2003), 1322-1328; ders.: Auswirkungen des eGovernment auf Verwaltungsstrukturen?, in: Henneke, H.-G. (Hg.): Künftige Funktionen und Aufgaben der Kreise im sozialen Bundesstaat, Stuttgart u. a., 2004, 163-178; s. auch Ernst, T.: Modernisierung der Wirtschaftsverwaltung durch elektronische Kommunikation, Köln u. a., 2005, 113 ff., 164ff.; Nolte, F.: E-Government in der Verwaltungsreform: Der große Sprung nach vorn?, in: Die Öffentliche Verwaltung, 22/60 (2007), 941-948; Schwiering, K.: Electronic Government: Ein Konzept zur innovativen Neugestaltung öffentlicher Aufgabenwahrnehmung, Münster, 2005; Voßkuhle, A.: Neue Verwaltungsrechtswissenschaften, in: Hoffmann-Riem, W./Schmidt-Aßmann, E./Voßkuhle, A. (Hg.): Grundlagen des Verwaltungsrechts, Band I, München, 2012, § 1 Rn. 65 ff.

$40 \mathrm{Zu}$ den verschiedenen Strategien der Länder Schliesky, U. (Hg.): eGovernment in Deutschland, Kiel, 2006, 21 ff.; zur Strategie E-Government 2.0 des Bundes Schallbruch, M.: E-Government 2.0 - Das Programm des Bundes, in: Zechner, A. (Hg.): Handbuch E-Government, Stuttgart, 2007, 23-28. 
über elektronische Medien“ versteht. ${ }^{41}$ Ein derartiges Begriffsverständnis ist aber zu weit, da so bereits der schlichte IT-Einsatz in der öffentlichen Verwaltung als E-Government zu qualifizieren wäre.

Vorzugswürdig erscheint insoweit der von der EU-Kommission zugrunde gelegte Ansatz: E-Government wird von dieser definiert als ,Einsatz der Informationsund Kommunikationstechnologien in öffentlichen Verwaltungen in Verbindung mit organisatorischen Änderungen und neuen Fähigkeiten, um öffentliche Dienste und demokratische Prozesse zu verbessern und die Gestaltung und Durchführung staatlicher Politik zu erleichtern“. ${ }^{42}$ Diesen Ansatz greift auch die Legaldefinition in $\S 2 \mathrm{Nr} .1$ EGovG $\mathrm{SH}^{43}$ auf; danach ist E-Government der Einsatz moderner Informations- und Kommunikationstechnik in öffentlichen Verwaltungen in Verbindung mit organisatorischen Veränderungen in den Geschäftsprozessen der öffentlichen Verwaltung zur Durchführung von Informations-, Kommunikations- und Transaktionsprozessen innerhalb und zwischen staatlichen Institutionen sowie diesen Institutionen und Bürgerinnen und Bürgern oder Unternehmen. So sperrig diese Legaldefinition auf den ersten Blick ist, so präzise wird auch deutlich, dass E-Government keine bloße „Elektrifizierung“ bestehender Verwaltungsstrukturen bedeutet, sondern zwangsläufig mit IT-bedingten Veränderungen der Organisation und des Verfahrens verbunden ist. ${ }^{44}$ Die Möglichkeiten der IT können nämlich erst dann effektiv und effizient

41 Von Lucke, J./Reinermann, H., in: Reinermann, H./von Lucke, J. (Hg.): Electronic Government in Deutschland, Speyer, 2002, 1 ff.; s. auch Hill, H.: eGovernment - Mode oder Chance zur nachhaltigen Modernisierung der Verwaltung, in: Bayerische Verwaltungsblätter, 24/134 (2003), 737-744.

42 Mitteilung der EU-Kommission „Die Rolle elektronischer Behördendienste (eGovernment) für die Zukunft Europas“, KOM (2003) 567 endg. vom 29. 09. 2003, 8; fortgeschrieben durch die Mitteilung der EU-Kommission „E-Government-Aktionsplan im Rahmen der i2010-Initiative - Beschleunigte Einführung elektronischer Behördendienste in Europa zum Nutzen aller", KOM (2006) 173 endg. vom 25. 04. 2006; diesem Ansatz folgend und auf die Verbesserung der Verwaltung abstellend Eifert, M., a. a. O., 21; Schliesky, U.: Verwaltungsreform als Aufgabe, a. a. O., 339 f.; Volkmann, U., a. a. O., Art. 91c Rn. 3; Voßkuhle, A., a. a. O., § 1 Rn. 65; ausführlich Schulz, S.E.: Der E-Government-Begriff der Europäischen Union: Die EU-Dienstleistungsrichtlinie als Chance für die Verwaltungsmodernisierung durch E-Government?, in: Verwaltung \& Management, 1/15 (2009), 3-12.

43 Gesetz zur elektronischen Verwaltung für Schleswig-Holstein (E-Government-Gesetz - EGovG) vom 08. 07. 2009, GVOB1. SH, 398.

44 Ebenso Britz, G.: Elektronische Verwaltung, a. a. O., § 26 Rn. 2; Eifert, M., a. a. O., 321 ff.; Schliesky, U.: Verantwortung im E-Government, a. a. O., 2; Volkmann, U., a. a. O., Art. 91c Rn. 3. - Die verfahrens- und organisationsrechtliche Problematik verschärft sich darüber hinaus durch die zunehmende Zeit- und Raumunabhängigkeit elektronischer Verwaltung, s. näher Boehme-Neßler, V.: Unscharfes Recht, 1. Aufl., Berlin, 2008, 112 ff.; Schliesky, U.: Raumbindung der Verwaltung in Zeiten des EGovernment, in: Schimanke, D. (Hg.): Verwaltung und Raum, 1. Aufl., Baden-Baden, 2009, 49-62; ders.: Einführung: Die Vermessung des virtuellen Raumes, in: Hill, H./Schliesky, U. (Hg.): Die Vermessung des virtuellen Raumes, 1. Aufl., Baden-Baden, 2012, 9-28. 
eingesetzt werden, wenn Organisation und Verfahren der Verwaltung an diesen Möglichkeiten ausgerichtet sind. Die IT verlässt insoweit die ihr immer wieder zugeschriebene dienende und instrumentelle Funktion, indem Anforderungen an Recht und Verwaltung formuliert werden. Kernprobleme von E-GovernmentProjekten sind heute regelmäßig nicht technischer, sondern rechtlicher Natur. ${ }^{45}$ Bei den aktuellen, an Örtlichkeit, Schriftlichkeit und Hierarchie ausgerichteten rechtlichen Vorgaben für Verwaltungsstrukturen stoßen E-GovernmentAnwendungen zwangsläufig an Grenzen, ${ }^{46}$ die überdacht und den tatsächlichen Möglichkeiten bei gleichzeitiger Wahrung fundamentaler Verfassungsprinzipien wie Rechtsstaat und Demokratie angepasst werden müssen. ${ }^{47} \mathrm{Zu}$ einer Weiterentwicklung des Verwaltungs- und ggf. auch des Verfassungsrechts besteht keine Alternative, will das Recht seinen Steuerungsanspruch aufrechterhalten ${ }^{48}$ und nicht Legitimitätsdefizite aufgrund eines zu großen Auseinanderklaffens der ITNutzung in Wirtschaft und Gesellschaft einerseits und der Verwaltung andererseits riskieren. Damit wird dann auch deutlich, dass E-GovernmentAnwendungen Verantwortungsstrukturen innerhalb der öffentlichen Verwaltung betreffen und so automatisch grundsätzliche Verantwortungsfragen aufwerfen, die einer verfassungsrechtlichen Klärung bedürfen - hier schließt sich der Kreis zu Art. 91c GG und weiteren Reformbedarfen.

Ein weiteres Kennzeichen des E-Government ist es, dass die elektronische Abwicklung von Verwaltungsvorgängen - durchaus unter Einschluss von Veränderungen der Verwaltungsorganisation und des Verwaltungsverfahrens regelmäßíg nicht auf rechtlicher Grundlage erfolgt. ${ }^{49}$ Hinsichtlich des Schweigens des Grundgesetzes zur IT in der öffentlichen Verwaltung konnte man bis

45 Umfassend dazu Kröger, D./Hoffmann, D. (Hg.): Rechts-Handbuch zum E-Government, Köln, 2005; Schliesky, U.: Über Begriff und Notwendigkeit einer staatlichen Innovationsmanagements, in: ders. (Hg.): Staatliches Innovationsmanagement, Kiel, 2010, 1-18, hier 9.

46 Am Beispiel des Grundgesetzes Schliesky, U.: Verfassungsrechtliche Rahmenbedingungen, a. a. O., 809 ff.; am Beispiel des Verfahrens- und Organisationsrechts ders.: Auswirkungen des E-Government, a. a. O., $322 \mathrm{ff}$; ders.: Verwaltungsmodernisierung und Verwaltungsverfahrensrecht, in: Mehde, V./Ramsauer, U./Seckelmann M. (Hg.): Staat, Verwaltung, Information, Festschrift für Hans Peter Bull zum 75. Geburtstag, Berlin, 2011, 819-840, hier 832 ff.

47 Schliesky, U. (Hg.): eGovernment in Deutschland, a. a. O., 5 ff.; ebenso Eifert, M., a. a. O., 28 ff.; Voßkuhle, A., a. a. O., § 1 Rn. 68.

48 Dazu Schliesky, U.: E-Government in Schleswig-Holstein: E-Government durch Recht, in: Zechner, A. (Hg.): Handbuch E-Government, Stuttgart, 2007, 49-54.

$49 \mathrm{Zu}$ einer entsprechenden Forderung des rechtlichen Nachvollziehens tatsächlich praktizierter Verwaltung Schliesky, U.: Regelungsbedarf für elektronische Verwaltungsstrukturen, in: Henneke, H.-G. (Hg.): Kommunale Verwaltungsstrukturen der Zukunft, Stuttgart u. a., 2006, 59-78; zur Relativierung des Rechts durch digitalisierte Medien eingehend Boehme-Neßler, V., a. a. O., $370 \mathrm{ff}$. 


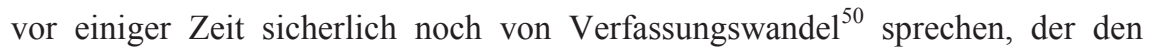
verfassungsrechtlichen Institutionen einen an gesellschaftlichen Realitäten ausgerichteten zeitgemäßen Inhalt gibt, doch sind die schon vorhandenen Änderungen so gravierend, dass eine derartige Erklärung sowohl den Begriff des Verfassungswandels als auch insbesondere die verfassungsrechtlichen Fundamente überdehnen würde. Denn - ohne dies hier vertiefen zu können ${ }^{51}$ - die Möglichkeiten der IT im Rahmen der Wahrnehmung von Verwaltungsaufgaben sowie das organisations- und verfahrensverändernde Verständnis von E-Government sind letztlich Generalangriffe auf die verfassungs- und verwaltungsrechtlichen Fundamente in Gestalt von (sachlicher und örtlicher) Zuständigkeit sowie Verwaltungsverfahren. Zuständigkeit und Verfahren sind verfassungsrechtliche Fundamentalinstitute, um rechtsstaatliche Verantwortung und demokratische Legitimation zu sichern. ${ }^{52}$ Würde man die Möglichkeiten des IT-Einsatzes in der öffentlichen Verwaltung konsequent zu Ende denken, so drohte die Auflösung von rechtsstaatlicher Verantwortung und demokratischer Legitimation in ,,vernetzter Beliebigkeit". ${ }^{33}$ Angesichts dieses verfassungsrechtlichen Zusammenhangs war es nur konsequent, im Zuge der Föderalismusreform II die IT in das Grundgesetz aufzunehmen.

Schließlich ist noch eine dritte Entwicklungslinie in den Blick zu nehmen, die ebenfalls durch die Möglichkeiten der IT forciert wird: Die Arbeitsteiligkeit, die in Wirtschaft und Gesellschaft schon längst Realität ist, erreicht nun in verstärktem Maße die Verwaltung. ${ }^{54}$ Dies hängt nicht nur mit der nach wie vor stark

$50 \mathrm{Zu}$ diesem Argumentationstopos näher Schliesky, U.: Souveränität und Legitimität, a. a. O., 455 ff.; s. auch Hain, K.-E., in: von Mangoldt, H./Klein, F./Starck, C. (Hg.): Kommentar zum Grundgesetz, Band 2: Art. 20-82, 6. Aufl., München, 2010, Art. 79 Rn. 13; Dreier, H., in: ders. (Hg.): Grundgesetz, Bd. 2, 2. Aufl., Tübingen, 1998, Art. 79 I, Rn. 37 ff.; Böckenförde, E.-W.: Anmerkungen zum Begriff des Verwaltungshandelns, in: Badura, P./Scholz, R. (Hg.): Wege und Verfahren des Verfassungslebens, Festschrift für Peter Lerche zum 65. Geburtstag, München, 1993, 3-14.; Bryde, B.-O.: Verfassungsentwicklung, 1. Aufl. Baden-Baden, 1982, 254 ff.; Schenke, W.-R.: Verfassung und Zeit, Archiv des öffentlichen Rechts, 4/103 (1978), 566-602, hier 585 ff.; Hesse, K.: Grenzen der Verfassungswandlung, in: Ehmke, H./Kaiser, J.H./Kewenig, W.A. u. a. (Hg.): Festschrift für Ulrich Scheuner zum 70. Geburtstag, Berlin, 1973, 123-141.

51 Dazu Eifert, M., a. a. O., 177 ff.; Schliesky, U.: Virtuelle Verwaltung? Auswirkungen moderner Kommunikationsmedien auf Verfahrensrecht und kommunale Verwaltungsstrukturen, in: Meyer, H/Wallerath, M. (Hg.): Gemeinden und Kreise in der Region, Stuttgart u. a., 2004, 80-111, hier $88 \mathrm{ff}$.

$52 \mathrm{Zu}$ diesem Zusammenhang Schliesky, U.: Souveränität und Legitimität, a. a. O., 230 ff.; ders.: Verfassungsrechtliche Rahmenbedingungen, a. a. O., $809 \mathrm{ff}$.

53 Schliesky, U.: Auswirkungen des E-Government, a. a. O., 1326.

54 Dazu eingehend Schliesky, U.: Legitimation und Verantwortung im komplexen, arbeitsteiligen Staat, in: Hill, H./Schliesky, U. (Hg.): Herausforderung e-Government, Baden-Baden, 2009, 11-38, hier 17 ff. 
zunehmenden Spezialisierung und Ausdifferenzierung von Fähigkeiten und Fertigkeiten, sondern auch mit Effizienzüberlegungen und daraus resultierenden Organisationsvorschlägen, wie einer Trennung zwischen Front Office und Back Office oder der Errichtung von sog. Shared-Service-Centern, zusammen. ${ }^{55} \mathrm{Zu}-$ dem etabliert sich auch in der Verwaltung zunehmend ein Prozessdenken, das als Business Process Management (BPM) einen wesentlichen Bestandteil des New Public Management (NPM) bildet und eine zentrale Voraussetzung für die Modellierung elektronischer Geschäftsprozesse darstellt. ${ }^{56}$ Gerade die IT-gestützte, arbeitsteilige Kooperation von Verwaltungen ist im Hinblick auf das sog. Verbot der Mischverwaltung allerdings äußerst problematisch: E-Government ist nach dem soeben Ausgeführten deutlich mehr als nur ein technisches Instrument der Aufgabenerledigung, sondern ein Organisation, Verfahren und Inhalt der Verwaltungsentscheidungen und damit die Ausübung von Staatsgewalt strukturierender, typisierender und koordinierender Verwaltungsmodus. ${ }^{57}$ Das Grundgesetz geht dagegen bislang von einem Trennprinzip bezüglich der Abschichtung von Kompetenzen und Verantwortungssphären aus. Dieses Aufgaben -und Kompetenzverständnis führt zu dem Grundsatz der vollständigen Aufgabenerledigung durch den jeweiligen Aufgabenträger selbst. ${ }^{58}$ Damit wird deutlich, dass erfolgreichen E-Government-Anwendungen der Vorwurf einer (verbotenen) Mischverwaltung inhärent ist.

\section{Informationsverarbeitung und Wissensgenerierung im Bundes- staat}

Die Bilanz nach vier Jahren Art. 91c GG dokumentiert, dass die Existenz der Verfassungsnorm und ihr intensiver Gebrauch durchaus Fortschritte in der föderalen IT-Steuerung erbrachte. Die zu Zeiten der Föderalismuskommission II noch zahlreichen Bedenkenträger sind nicht mehr $\mathrm{zu}$ vernehmen. Trotz des Kompromisscharakters konnte die Vorschrift die Erwartungen also erfüllen und

55 Dazu eingehend Schulz, E.: One-Stop Government, Kiel, 2007, 15 ff., 49 ff.; Tallich, M.: Shared Service Center als innovative Organisationsform, Kiel, 2012.

56 Dazu Ziekow, J.: Vom Verwaltungsverfahren über den Geschäftsprozess zum IT-Workflow, in: Hill, H./Schliesky, U. (Hg.): Herausforderung e-Government, Baden-Baden, 2009, 69-87.

57 Ruge, K., a. a. O., Art. 91c Rn. 3; Schliesky, U.: E-Government - Schlüssel zur Verwaltungsmodernisierung oder Angriff auf bewährte Verwaltungsstrukturen?, in: Landes- und Kommunalverwaltung, 3/27 (2005), 89-95, hier 90 f.; ders.: Legitimation und Verantwortung, a. a. O., 19 ff.; Volkmann, U., a. a. O., Art. 91c Rn. 4.

58 BVerfGE 119, 331 (367). 
den ebenenübergreifenden Einsatz von Informations- und Kommunikationstechnik in Deutschland voranbringen.

Allerdings stellt sich nun die Frage, ob der erreichte Zustand - gerade mit Blick auf künftige Herausforderungen - bereits ausreichend ist und Handlungsbedarf nunmehr ausschließlich auf einfachgesetzlicher bzw. gar verwaltungstatsächlicher Ebene zu verorten ist, oder ob die schon jetzt absehbaren Herausforderungen der Informations- und Kommunikationstechnik auch weiteren verfassungsrechtlichen Normierungsbedarf auslösen. Diese Frage soll nachfolgend am Themenkomplex „Informationsverarbeitung und Wissensgenerierung“ untersucht werden. Auch wenn die Charakterisierung von Staat und Gesellschaft als Informations- und/oder Wissensgesellschaft ${ }^{59}$ (,technisierte Informationsgesellschaft" $)^{60}$ verfassungsrechtlich zunächst irrelevant ist, wie es auch die Einordnung als „Industriegesellschaft“ im 20. Jahrhundert war, so steht der Staat doch unzweifelhaft vor einer großen Herausforderung, seine Rolle in dieser soziologisch beschriebenen Gesellschaft zu finden und seinen klassischen als auch neuen Aufgaben in diesem Kontext gerecht werden zu können. Schlagwörter wie Informationsfreiheit, Transparenz, Open Data bzw. Open Government Data finden sich zur Kennzeichnung dieser neuen Anforderungen, zudem stehen Informationsverarbeitung und Wissensgenerierung im Bundesstaat zunehmend im politischen wie rechtlichen Fokus. Mit gutem Grund legt daher der ITPlanungsrat in seiner nationalen E-Government-Strategie einen Schwerpunkt auf die Themen Information und Kommunikation. Ein Leitprojekt des Rates, das vom Bundesministerium des Innern und dem Ministerium der Finanzen in Sachsen-Anhalt federführend betreut wird, widmet sich dem „Föderalen Informationsmanagement". 61

59 Dazu grundlegend Luhmann, N.: Die Gesellschaft der Gesellschaft, 1. Aufl., Frankfurt a. M., 1997/1998, 1088 ff., 1106; Sonntag, P. (Hg.): Die Zukunft der Informationsgesellschaft, Frankfurt a. M., 1983; s. auch Albers, M.: Die Komplexität verfassungsrechtlicher Vorgaben für das Wissen der Verwaltung, in: Spiecker gen. Döhmann, I./Collin, P. (Hg.): Generierung und Transfer staatlichen Wissens im System des Verwaltungsrechts, Tübingen, 2008, 50-72; Schulz, S.E.: E-Daseinsvorsorge - der staatliche und kommunale Grundversorgungsauftrag in einer technisierten Informationsgesellschaft, in: Schliesky, U. (Hg.): Selbstverwaltung im Staat der Informationsgesellschaft, Kiel, 2010, 101-128, hier $112 \mathrm{ff}$.

60 BVerfGE 125, 175 (224); dazu Schulz, S.E.: Informations- und Kommunikationstechnologie als Grundversorgung, in: Datenschutz und Datensicherheit, 10/34 (2010), 698-702.

61 Bundesministerium des Innern in Zusammenarbeit mit dem Ministerium der Finanzen Sachsen-Anhalt (Hg.): FIM - E-Government mit Zukunft, 2012. S. auch www.it-planungsrat.de/fim. 


\section{Begrifflichkeit}

Zunächst einmal muss geklärt werden, was unter der „Verarbeitung von Informationen“ und der „Generierung von Wissen“ zu verstehen ist. Der Blick in das Grundgesetz zeigt ohne Weiteres, dass „Information“ und „Wissen“ keine verfassungsrechtlichen Begriffe sind. Wohl gewährleistet Art. 5 Abs. 1 S. 1 GG auch die Informationsfreiheit und damit das Recht, sich selbst zu informieren, als eigenständiges Grundrecht. ${ }^{62}$ Geschützt ist sowohl das aktive Handeln zur Informationsbeschaffung als auch die schlichte Entgegennahme von Informationen. ${ }^{63}$ Dieser grundrechtliche Ansatz ist für den Kontext des Föderalismus und damit für das Staatsorganisationsrecht jedoch unergiebig.

Für die dennoch erforderliche Begriffsklärung ist daher eine allgemeinere Ebene aufzusuchen. Die kleinste Einheit und Vorstufe für Information und Wissen sind zunächst Daten. In Anlehnung an die Systemtheorie lassen sich Daten als ,beobachtbare Unterschiede“ beschreiben. ${ }^{64}$ Heutzutage wird der Begriff „Daten“ in der Regel in Verbindung mit Informations- und Kommunikationstechniken verwendet. In diesem Kontext bezeichnen Daten ,Zeichen oder Zeichenketten, die aufgrund von bekannten oder unterstellten Vereinbarungen Informationen darstellen und zum Zwecke der Verarbeitung im Computer gespeichert werden“. 65

Aus den Daten entstehen Informationen; sie sind gekennzeichnet durch einen Inhalt, einen aufnehmbaren Bedeutungsgehalt und eine systembedingte Verarbeitungsfähigkeit. Informationen sind in einer bestimmten Weise aufbereitete Daten und damit Vorprodukte und zugleich zwingende Voraussetzungen von Wissen. ${ }^{66}$ Aus diesen Informationen kann - in der Regel vor allem mit Hilfe intellektuellen Bemühungen, aber auch mit Hilfe moderner Informations- und Kommunikationstechnik - Wissen entstehen. Wissen ist verarbeitete und in einen Kontext gestellte Information als Basis für Entscheidungen und Handlungen. ${ }^{67}$ Wissen

62 Antoni, M., in: Hömig, D. (Hg.): Grundgesetz, 10. Aufl., Baden-Baden, 2013, Art. 5 Rn. 9; Herzog, R., in: Maunz, T./Dürig, G. (Begr.), Grundgesetz, 67. Erg.-Lfg., München, 2012, Art. 5 Rn. 81.

63 BVerfGE, 27, 71 (82 f., 108 f.); 103, 44 (60 ff.)

64 Willke, H., Einführung in das systemische Wissensmanagement, Heidelberg, 2004, 28 f.; diesem folgend Kluth, W.: Die Strukturierung von Wissensgenerierung durch das Verwaltungsorganisationsrecht, in: Spiecker gen. Döhmann, I./Collin, P. (Hg.): Generierung und Transfer staatlichen Wissens im System des Verwaltungsrechts, Tübingen, 2008, 73-92, hier 75.

65 So die Legaldefinition in $\S 2$ Nr. 3 EGovG SH.

66 S. Kluth, W.: Die Strukturierung von Wissensgenerierung, a. a. O., 76; vgl. auch IT-Wissen, Das große Onlinelexikon für Informationstechnologie, Art. Information, abgerufen am 22.02.2013.

67 Kluth, S.: Die Integrationsverantwortung der Landesparlamente, in: Landes- und Kommunalverwaltung, 7/20 (2010), 302-306, hier 304. 
entsteht somit, wenn Informationen in einen Praxiszusammenhang eingebunden werden und daraus eine neue oder eine veränderte Praxis folgt. ${ }^{68}$

Gerade im Hinblick auf Informations- und Kommunikationstechnik bestehen Informationen aus Daten, die in IT-Systemen automatisiert verarbeitet werden, als Informationen abrufbar sind und zumindest dabei helfen, Wissen entstehen zu lassen. Die Informationsverarbeitung folgt - vor allem im technischen Kontext, aber nicht zwingend - der Datenverarbeitung, die letztlich die Verwendung bzw. den Umgang mit Daten meint. ${ }^{69}$ Die Informationen werden bearbeitet, verwendet, in einen bestimmten Kontext gestellt, um als nächste „Veredelungsstufe“ Wissen zu generieren, d. h. zu schaffen. Wissen wiederum setzt einen Erkenntnisprozess voraus, der zwar durchaus mithilfe automatisierter Prozesse durchgeführt werden kann, letztlich aber ohne den menschlichen Intellekt, der nämlich mindestens diese Prozesse programmieren muss, und ohne menschliches Bewusstsein nicht möglich ist. Prozesshaft beschreibt der Terminus „Wissensgenerierung“ den gesamten Vorgang von der Isolierung und Erhebung der Daten über die Informationsaufbereitung bis hin zur Schaffung von Wissen, ${ }^{70}$ das dann zwingende Voraussetzung von rationalen staatlichen Entscheidungen ist.

\section{Verfassungsrechtliche Relevanz}

Wie diese Informationsverarbeitung und Wissensgenerierung im staatlichen Bereich zu erfolgen hat, ist durch das Verfassungs- und Verwaltungsrecht vorgegeben. Bestimmende Faktoren sind vor allem die Aufbauorganisation ${ }^{71}$ und die Ablauforganisation in Gestalt von Verfahrensrecht und praktizierten Verfahrensmustern. ${ }^{72}$ Die Bedeutung von Informationsverarbeitung und Wissensgenerierung liegt also vor allem in den rechtlich determinierten Entscheidungsprozessen, die letztlich eine staatliche Entscheidung und damit die Ausübung von Staatsgewalt zum Ziel haben. ${ }^{73}$ Es existiert somit durchaus ein föderales System der Informationsverarbeitung und Wissensgenerierung, das in Ansätzen eine

68 Kluth, W.: Die Strukturierung von Wissensgenerierung, a. a. O., 76.

69 S. etwa $\S 2$ Abs. 2 S. 1 LDSG SH: „Datenverarbeitung ist die Verwendung personenbezogener Daten.“

70 Kluth, W.: Die Strukturierung von Wissensgenerierung, a. a. O., 78.

$71 \mathrm{Zu}$ den verschiedenen Organisationstypen: Schuppert, G.F.: Verwaltungswissenschaft, 1. Aufl., BadenBaden, 2000, $579 \mathrm{ff}$.

72 Kluth, W.: Die Strukturierung von Wissensgenerierung, a. a. O., 78.

73 Das Bundesverfassungsgericht definiert den Begriff der „Staatsgewalt“ i. S. d. Art. 20 GG bekanntlich als ,jedenfalls alles amtliche Handeln mit Entscheidungscharakter“, s. BVerfGE 83, 60 (73); 93, 37 (37, $68)$. 
verfassungsrechtliche Abbildung vor allem durch die Ordnung der Gesetzgebungs- und Verwaltungskompetenzen gefunden hat. Als Neuerung in diesem System sei auf Art. 91c GG hingewiesen, der vor dem Hintergrund des Dogmas eines „Verbots der Mischverwaltung“ in ersten Ansätzen hilft, Kompetenzgrenzen bei der Informationsverarbeitung zu überwinden.

Information und Wissen sind Voraussetzungen der Ausübung von Staatsgewalt. Sie haben eine erhebliche Relevanz für staatliche Entscheidungsprozesse ${ }^{74}$ und sind daher verfassungsrechtlich an den Vorgaben für die Kreierung und Ausübung von Staatsgewalt in Gestalt des Gebotes demokratischer Legitimation der Staatsgewalt zu messen. Staatsgewalt erscheint nicht plötzlich in der Realität, sondern ist vor allem in ihrer Form als Entscheidung das Ergebnis eines Meinungsbildungs-, Willensbildungs- und Entscheidungsprozesses. Das Grundgesetz schweigt zu den Einzelheiten dieser Prozesse ebenso wie zu den Begriffen „Information“ und „Wissen“. Das rechtsstaatliche Entscheidungsverfahren lässt sich in verschiedene Bestandteile ausdifferenzieren, die unterschiedlichen Anforderungen demokratischer Legitimation unterliegen. ${ }^{75}$ Jeder Entscheidungsvorgang lässt sich insoweit in die Bestandteile „Meinungsbildung“ und „Willensbildung“ untergliedern, wobei die Willensbildungsphase - gerade bei Kollegialorganen oder dem Zusammenwirken verschiedener Behörden - wiederum aus einer Beratungs- und einer (eigentlichen) Entscheidungsphase besteht. ${ }^{76}$ Am Anfang steht die Meinungsbildung, in der die für den Entscheidungsprozess erforderlichen Informationen zusammengetragen werden. Hier finden die - in der Regel öffentlichen - Diskussionen statt, in denen Anregungen und Vorschläge für Entscheidungsnotwendigkeit und Entscheidungsinhalt unterbreitet sowie Stellungnahmen zu bestimmten Problemstellungen abgegeben werden. Informationen werden gesammelt, aufbereitet und verarbeitet. Der Legitimationsbedarf ist hier aufgrund der großen Distanz zur Entscheidung regelmäßig am geringsten. ${ }^{77}$

In der Willensbildungsphase bilden die zur Entscheidung berufenen Organe aufgrund der zuvor erarbeiteten Meinungen einen (organschaftlichen) Willen. In

74 Ausführlich zur Rolle von Information und Wissen für staatliche Innovationsprozesse Schulz, S.E: Wissensmanagement als Basis staatlicher Innovation, in: Hill, H./Schliesky, U. (Hg.): Innovationen im und durch Recht - E-Volution des Rechts- und Verwaltungssystems II, Baden-Baden, 2010, 249-283.

75 Dazu näher BVerfGE 8, 104 (104 Ls. 6; 114 f.); Jochum, G.: Materielle Anforderungen an das Entscheidungsverfahren in der Demokratie, Berlin, 1997, S. 20 f.

76 Hierzu im Folgenden Schliesky, U.: Souveränität und Legitimität, a. a. O., 696 f.

77 Zur Entscheidungsnähe als maßgebliches Kriterium auch Schulz, S.E.: Vernetztes Wissen - Shared Services im Wissensmanagement, in: Schliesky, U./Schulz, S.E. (Hg.): Die Erneuerung des arbeitenden Staates, 1. Aufl., Baden-Baden, 2012, 113-140, hier 136 f. 
der Willensbildungsphase muss nun auch auf staatlich verbindliches Wissen zurückgegriffen werden, das als richtig und verbindlich von den Organwaltern vorausgesetzt werden muss, um zu einer rechtmäßigen Entscheidung zu gelangen. Im ersten Teil der Willensbildungsphase, der Beratungsphase, werden die zuvor gesammelten Informationen staatlich verbindlich „verarbeitet“. Unter Abwägung der verschiedenen beteiligten Interessen kristallisiert sich der dem Organ zugerechnete Wille, d. h. Entscheidungsinhalt, durch Diskussionen der zuvor gebildeten Meinung heraus. Im zweiten Teil, der eigentlichen Entscheidungsphase, trifft das nach der Kompetenzordnung dazu berufene Herrschaftsorgan dann die verbindliche und nach außen wirkende Entscheidung, die den im Verlauf der Beratungsphase gebildeten Willen, die Maßnahmeintention, rechtlich verbindlich fixiert. Je näher diese eigentliche Entscheidung rückt, desto problematischer und grundsätzlich geringer werden die Mitwirkungsmöglichkeiten von personell nicht legitimierten Akteuren.

An die Entscheidung schließt sich die Vollzugsphase an, in der die Entscheidung umgesetzt wird. Wie dies zu erfolgen hat, hängt von der Art der Entscheidung etwa ein Gesetz, ein Verwaltungsakt oder die bloße Bekanntgabe einer Entscheidung über Informationen oder Wissen - ab.

Informationen und Wissen sind somit zum einen die Voraussetzungen für staatliche Entscheidungen; Informationsverarbeitung und Wissensgenerierung haben Einfluss auf die Rechtmäßigkeit und die Legitimität staatlicher Entscheidungen. Dabei kann allerdings - aufgrund der Komplexität und der erst zunehmenden Verbindlichkeit dieses staatlichen Entscheidungsprozesses - nicht holzschnittartig eine bestimmte Anforderung an den Legitimationsbedarf oder das genaue Procedere der Gewinnung von Information und Wissen gestellt werden. Zum anderen kann eine staatliche Maßnahme - und dies geschieht zunehmend häufiger - gerade die Übermittlung bzw. Veröffentlichung von Informationen und/oder Wissen zum Gegenstand haben. Die bekannten Beispiele staatlicher Warnungen und Empfehlungen fallen ebenso in diese Kategorie wie Maßnahmen nach Informationsfreiheitsgesetzen oder nach dem Informationsweiterverwendungsgesetz. In beiden Fällen gilt, dass die vorgeschriebenen Zuständigkeiten und Verfahren der Informationsverarbeitung und Wissensgenerierung durchlaufen werden, die sich vor allem als verwaltungsrechtliche Konkretisierungen finden, ${ }^{78}$ vereinzelt aber auch unmittelbar im Grundgesetz normiert sind. ${ }^{79}$ 
Es ist das Verdienst des Informationsrechts, die verfassungsrechtlichen Vorgaben für Informationsverarbeitung und Wissensgenerierung herausgearbeitet zu haben. ${ }^{80}$ Angesichts dieser für das Grundgesetz bislang unbekannten Perspektive bedarf es keiner ausführlichen Begründung, dass aus Sicht eines informationsrechtlichen Ansatzes ausführliche und stimmige Regelungen vergeblich zu suchen sind. Die verfassungsrechtlichen Vorgaben für Informationen und Wissen sind nur durch Auslegung zugänglich, so wie es das Bundesverfassungsgericht in allerdings umstrittener Art und Weise - in seiner Glykolwein-Entscheidung bekanntlich praktizierte. Dort hat das Gericht entschieden, dass die Bundesregierung aufgrund ihrer Aufgabe der Staatsleitung überall dort zum Handeln berechtigt ist, wo ihr eine gesamtstaatliche Verantwortung zukommt und diese durch Informationstätigkeit wahrgenommen werden kann. ${ }^{81}$ Für diese Informationstätigkeit des Staates gilt insoweit als verfassungsrechtliche Vorgabe, dass eine staatliche Aufgabe vorliegen muss, die Zuständigkeitsordnung eingehalten wird und die Anforderungen an Richtigkeit und Sachlichkeit der Information beachtet werden. ${ }^{82}$ Letztere Kriterien stellen Voraussetzungen für Informationsverarbeitung und Wissensgenerierung dar. Das Bundesverfassungsgericht betont mit Recht, dass sich die staatliche Teilhabe an öffentlicher Kommunikation in einem fortlaufenden Entwicklungsprozess befindet, der durch die heutigen Massenmedien, die Informations- und Kommunikationstechnik und neue Informationsdienste geprägt wird. ${ }^{83}$ Das Gericht konstatiert ebenfalls zu Recht eine Erwartungshaltung der Bevölkerung und betont, ein Schweigen der Regierung würde zu Legitimationsverlusten führen. ${ }^{84} \mathrm{Da}$ die Vollständigkeit einer Information ein wichtiges Element der Glaubwürdigkeit staatlichen Handelns darstelle, sei die problemangemessene und ggf. Kompetenzen anderer Staatsorgane überschreitende Unterrichtung durch die Bundesregierung unter dem Aspekt der föderalen Kompetenzaufteilung unbedenklich. ${ }^{85}$ So pragmatisch dieser Ansatz auch ist, so

80 Dazu grundlegend Kloepfer, M.: Informationsrecht, München, 2002, § 3 Rn. 1 ff.; zu materiellen informationsbezogenen Verbürgungen des Staatsorganisationsrechts ebd., § 3 Rn. $106 \mathrm{ff}$.

81 BVerfGE 105, 279 ff. (Osho); 105, 252 ff. (Glykol); ausführlich zu diesem Themenkomplex, insbesondere auch zu neueren Entwicklungen, Manssen, G., in: von Mangoldt, H./Klein, F./Starck, C. (Hg.): Kommentar zum Grundgesetz, Band 1, 6. Aufl., München, 2010, Art. 12 Rn. 86 ff. m. w. N. Zuletzt Geldermann, J./Hammer, T.: Gesetzgebungskompetenzen als Grenze der Verbraucherinformation durch den Bund, in: Verwaltungsarchiv, 1/104 (2013), 64-82.

82 BVerfGE 105, 252 (268, 272 f.).

83 BVerfGE 105, 252 (268 f.).

84 BVerfGE 105, 252 (270).

85 BVerfGE 105, 252 (271 f.). 
ratlos lässt er den Betrachter angesichts des zuvor dargestellten Zusammenhangs der Informationsverarbeitung und Wissensgenerierung zur Ausübung von Staatsgewalt und damit einem potentiellen Bedürfnis nach demokratischer Legitimation zurück. $^{86}$

\section{Föderaler Informationsverbund als Netzwerk}

Die Probleme verschärfen sich nun durch die elektronischen Möglichkeiten der Informationsverarbeitung und Wissensgenerierung, die den verfassungsrechtlich vorgesehenen linearen Wegen der Wissensgenerierung und Entscheidungsfindung nicht mehr entsprechen. Die vielfältigen - vor allem gemeinschaftsrechtlichen - Pflichten zum Informationsaustausch und zur Zusammenarbeit in einem elektronischen Behördennetz haben längst eine Netzwerkverwaltung Realität werden lassen. Nicht zuletzt Art. 28 EU-DLR, ${ }^{87}$ aber auch zahlreiche weitere Rechtsakte - zuletzt bis hin zum Kommissionsvorschlag zur Vernetzung der Arbeitsverwaltungen $^{88}$ - haben ein solches europäisches Behördennetz zum Informationsaustausch und zur Wissensgenerierung errichtet.

Der Begriff des Netzwerks gibt dabei - jedenfalls bislang - keine neuen Maßstäbe für Inhalt oder Zustandekommen einer Entscheidung vor, bildet aber eine Kategorie zur organisatorischen bzw. institutionellen Beschreibung von komplexen Verwaltungsentscheidungen und kann insoweit zur Rationalität und vielleicht auch zur Optimierung von Entscheidungsprozessen dienen. ${ }^{89}$ In dem Moment, in dem der Gesetzgeber das Netzwerk aber zum Rechtsbegriff macht, muss diese Gleichgültigkeit einem normativen Konzept weichen. Und Beispiele einer juristischen Verwendung finden sich inzwischen zahlreich: $§ 50 \mathrm{a}$ GWB ist in der

86 Für das Handeln der Exekutive - und nicht Regierungshandeln - ist die maßgebliche Rechtsfrage, ob und inwieweit die hoheitliche Information als Handlungsform in Grundrechtspositionen eingreift und aus diesem Grunde dem Gesetzesvorbehalt unterliegt, also nur auf der Grundlage einer formellen Rechtsgrundlage zulässig ist; exemplarisch zu diesem Problemkreis Holzner, T.: Die Pankower Ekelliste, in: Neue Zeitschrift für Verwaltungsrecht, 8/29 (2010), 489-494; Schoch, F.: Amtliche Publikumsinformation zwischen staatlichem Schutzauftrag und Staatshaftung, in: Neue Juristische Wochenschrift, 39/65 (2012), 2844-2850; Becker, F./Blackstein, Y.: Der transparente Staat - Staatliche Verbraucherinformationen über das Internet, in: Neue Juristische Wochenschrift, 8/64 (2011), 490-494.

87 Dazu näher Schliesky, U.: Die Europäisierung der Amtshilfe, Stuttgart u. a., 2008, 38.

88 Mitteilung der EU-Kommission „Reform des europäischen Beschäftigungsnetzes EURES“ vom 26. 11. 2012.

89 Boysen, S. u. a.: Netzwerke im Öffentlichen Recht, in: dies. u. a. (Hg.): Netzwerke, 1. Aufl., BadenBaden, 2007, 289-300, hier 299. 
amtlichen Überschrift mit „Zusammenarbeit im Netzwerk der europäischen Wettbewerbsbehörden" betitelt. ${ }^{90}$

Das Netzwerk ist eher eine Herausforderung an das Verfahrens- als an das Organisationsrecht; beide Materien sollten allerdings nicht so strikt getrennt, wie bislang in der deutschen Dogmatik geschehen, behandelt werden. Denn die Arbeitsteilung im europäischen wie im nationalen Behördennetz betrifft nicht mehr nur sog. „Verwaltungsdienstleistungen“ ohne Entscheidungscharakter ${ }^{91}$ sondern zunehmend auch klassische Entscheidungsprozesse. Ließe sich ein solcher Entscheidungsprozess bei dem monistischen Legitimations- und Entscheidungsansatz bislang als eindimensionales Verfahren der Informationsgewinnung, -verarbeitung, Diskussion, Meinungsbildung-, Willensbildungs- und Entscheidungsphase verstehen, ${ }^{92}$ so ordnet das Recht zunehmend mehrdimensionale Entscheidungsprozesse im Netzwerk an, die angesichts unterschiedlicher Regelungen der Informationsgewinnung, unterschiedlicher rechtlicher Rahmenbedingungen für die Ausübung der konkreten Sachzuständigkeit und des Bedürfnisses nach einer sauberen Abgrenzung sowie Verzahnung von Verantwortungssphären einer normativen Abbildung bedürfen. Dies betrifft auch Netzwerke, in denen „nur“ Informationen ausgetauscht werden, da angesichts der Geltung des Amtsermittlungsgrundsatzes gem. § $24 \mathrm{VwVfG}$ bei gleichzeitig steigenden Wissensdefiziten der öffentlichen Verwaltung angesichts immer komplexerer Entscheidungsgrundlagen die Informations- und daraus resultierende Handlungsverantwortung zunehmend an Bedeutung und insbesondere Grundrechtsrelevanz gewinnt. Das Prozessdenken und damit die Modularisierung von Verwaltungsverfahren und Informationsverarbeitungs- wie Entscheidungsprozessen sind erforderlich, um das Netzwerk normativ überhaupt sichtbar und damit steuerund beherrschbar zu machen. Damit erweist es sich dann auch als wichtiges

90 Vgl. zu §50a GWB Rehbinder, E., in: Immenga, U./Mestmäcker, E.-J. (Hg.): Wettbewerbsrecht, 4. Aufl., München, 2007, § 50a GWB, Rn. 1 ff.

91 Die oftmals irreführend als „Querschnittsaufgaben“ bezeichnet werden und die sich gerade zur Wahrnehmung in „modernen“ Organisationsformen wie Shared Services Center eigneten. Zu denken ist dabei vor allem an Dienstleistungen, die die Merkmale ,hohe Standardisierbarkeit“, großes Volumen (economies of scale) und „Erfordernis spezieller Kenntnisse“ erfüllen (Schütz, G.: Wirksamkeit und Wirtschaftlichkeit der Bundesverwaltung durch „Shared-Service-Center“ („SSC“), in: Hill, H. (Hg): Die Zukunft des öffentlichen Sektors, 1. Aufl., Baden-Baden, 2005, 23-36, hier 28). Exemplarisch lassen sich hier gemeinsame Liegenschaftsverwaltung (neudeutsch: Gebäude- oder Facility-Management), zentrale Personalverwaltung, Justitiariat oder der Betrieb von unterschiedlichen Körperschaften genutzten Rechenzentren nennen - diese lassen sich jedoch zutreffend als „operative Hilfstätigkeiten“ betiteln, um den fehlenden Entscheidungscharakter zu betonen.

92 Dazu eingehend Schliesky, U.: Souveränität und Legitimität, a. a. O., 245 f., 696 f. 
Instrument zur Koordinierung von Interessen bei der Abwägung im Verwaltungsrecht, indem es eine dienende Funktion für einen kommunikativen Ausgleich von Interessenkonflikten im Rahmen von Entscheidungsspielräumen der Verwaltung bekommen kann. ${ }^{93}$ Für die normative Erfassung sollte dann auch terminologisch zwischen Netzwerken - unter Einbeziehung privater Akteure und Behördennetzen - der Teilmenge der ausschließlich dem staatlichen Sektor zugehörigen Behörden - differenziert werden.

Und schließlich - drittens - muss das Verbot der sog. Mischverwaltung in die Betrachtungen einbezogen werden: Die Arbeitsteiligkeit, die in Wirtschaft und Gesellschaft schon längst Realität ist, erreicht nun in verstärktem Maße die Verwaltung. Dies hängt nicht nur mit der nach wie vor stark zunehmenden Spezialisierung und Ausdifferenzierung von Fähigkeiten und Fertigkeiten, sondern auch mit Effizienzüberlegungen und daraus resultierenden Organisationsvorschlägen wie einer Trennung zwischen Front Office und Back Office oder der Errichtung der angesprochenen Shared Service Center zusammen. ${ }^{94}$

Genau hier muss allerdings der Blick präzise auf die verfassungsrechtliche Ausgangslage gerichtet werden - und dieser Blick löst in Bezug auf die Vorstellungen von einer modernen Verwaltungswelt Erschütterung aus. Denn das Grundgesetz geht erkennbar von einem Trennungsprinzip hinsichtlich der Abschichtung von Kompetenzen und Verantwortungssphären aus. Dieses Aufgaben- und Kompetenzverständnis führt zu einem Grundsatz der vollständigen Aufgabenerledigung durch den jeweiligen Aufgabenträger selbst. Das Bundesverfassungsgericht hat dies in seiner sog. „Hartz IV“-Entscheidung zu der verfassungsrechtlichen Zulässigkeit der Arbeitsgemeinschaften gem. § 44b SGB II noch einmal deutlich herausgearbeitet. ${ }^{95}$ Das Gericht postuliert zunächst ein grundsätzlich

93 Boysen, S., a. a. O., 291.

94 Dazu statt vieler Schulz, E.: One-Stop-Government, a. a. O., 15 ff., 49 ff.; Lietz, G.: Shared Services E-Government und Economies of Scale, in: Zechner, A. (Hg.): Handbuch E-Government, Stuttgart, 2007, 269-278; zum Aspekt der Arbeitsteiligkeit Schliesky, U.: Die Aufnahme der IT in das Grundgesetz, a. a. O., 318 ff.; ders.: Legitimation und Verantwortung, a. a. O., 11 ff. i. E.; zum Shared-ServiceGedanken Ruge, K.: Verwaltungsmodernisierung durch E-Government, in: Niedersächsische Verwaltungsblätter, 3/15 (2008), 89-94, hier 91 f.; Schütz, G.: Wirksamkeit und Wirtschaftlichkeit, a. a. O. 23 ff.; Maier, H./Gebele, B.: Shared Service Center und das Ressortprinzip des Art. 65 S. 2 GG, in: Deutsche Verwaltungspraxis, 7/122 (2007), 270-278.

95 BVerfGE 119, 331 ff.; dazu Peters, K.: Bundesverfassungsgericht erklärt ARGEn für verfassungswidrig, in: Nachrichtendienst, 2/88 (2008), 53-54; Meyer, H.: Das SGB II als Ernstfall des Föderalismus, in: Neue Zeitschrift für Verwaltungsrecht, $3 / 27$ (2008), 275-278; zum angesprochenen „Verbot der Mischverwaltung“ Waldhoff, C.: Titel, in: Zeitschrift für Staats- und Europawissenschaften, 1/6 (2008), 57-74; Trapp, D.B.: Die Kontinuität der bundesverfassungsgerichtlichen Rechtsprechung zur Mischverwaltung, in: Die Öffentliche Verwaltung, 7/61 (2008), 277-282; Schnapp, F.: Mischverwaltung im Bundesstaat 
striktes Trennungsprinzip hinsichtlich der Verwaltungskompetenzen: „Die Verwaltungszuständigkeiten von Bund und Ländern sind grundsätzlich getrennt und können selbst mit Zustimmung der Beteiligten nur in den vom Grundgesetz vorgesehenen Fällen zusammengeführt werden. Zugewiesene Zuständigkeiten sind mit eigenem Personal, eigenen Sachmitteln und eigener Organisation wahrzunehmen. Ausnahmen hiervon sind nur in seltenen Fällen und unter engen Voraussetzungen zulässig“ “. ${ }^{96}$ Aus einem derartigen Kompetenzverständnis leitet das Gericht nicht nur das sog. Verbot der Mischverwaltung ab, ${ }^{97}$ sondern formuliert auch einen Grundsatz der vollständigen Aufgabenerledigung durch den jeweils zuständigen Verwaltungsträger selbst: „Der Verwaltungsträger, dem durch eine Kompetenznorm des Grundgesetzes Verwaltungsaufgaben zugewiesen worden sind, hat diese Aufgaben grundsätzlich durch eigene Verwaltungseinrichtungen, also mit eigenem Personal, eigenen Sachmitteln und eigener Organisation wahrzunehmen (...) Von dem Gebot, die Aufgaben eigenverantwortlich wahrzunehmen, darf nur wegen eines besonderen sachlichen Grundes abgewichen werden.“98 Das Bundesverfassungsgericht sieht diese klare Zuordnung von Verwaltungszuständigkeiten als eine Frage des Demokratieprinzips an. Nach Auffassung des Gerichts fehlt es an dem erforderlichen Niveau demokratischer Legitimation, wenn ,die Aufgaben durch Organe oder Amtswalter unter Bedingungen wahrgenommen werden, die eine klare Verantwortungszuordnung nicht ermöglichen. Der Bürger muss wissen können, wen er wofür - auch durch Vergabe oder Entzug seiner Wählerstimme - verantwortlich machen kann““ ${ }^{99}$ Das Gericht macht nebenbei schließlich auch deutlich, dass IT-Fragen offenbar kein rechtfertigender Grund für Ausnahmen von den eben dargestellten Grundsätzen

nach der Föderalismusreform, in: Jura, 4/30 (2008), 241-244; Burgi, M.: Zukunftsfähige Kooperationen trotz Entflechtung und statt Hochzonung, in: Zeitschrift für Staats- und Europawissenschaften, 2/6 (2008), 281-303; im Zusammenhang mit der in Schleswig-Holstein diskutierten Übertragung der Aufgaben des Einheitlichen Ansprechpartners auf eine Anstalt in gemeinsamer Trägerschaft von Land, Kommunen, Industrie- und Handelskammern sowie Handwerkskammern Luch, A.D./Schulz, S.E.: Wahrnehmung der Aufgaben des Einheitlichen Ansprechpartners nach Art. 6 der EUDienstleistungsrichtlinie durch eine Anstalt des öffentlichen Rechts in gemeinsamer Trägerschaft von Land, Kommunen und Kammern, in: Die Gemeinde Schleswig-Holstein, 5/60 (2008), 118-125; s. auch Schulz, S.E.: Kooperationsmodelle zur Umsetzung des Einheitlichen Ansprechpartners als unzulässige Mischverwaltung?, in: Die Öffentliche Verwaltung, 24/61 (2008), 1028-1036.

96 BVerfGE 119, 331 (364).

97 BVerfGE 119, 331 (365); vgl. ferner BVerfGE 63, 1 (38 ff.); 108, 169 (182); Schulz, S.E.: Kooperationsmodell, a. a. O., $116 \mathrm{ff}$.

98 BVerfG, 119, 331 (367).

99 BVerfGE 119, 331 (366). 
sein sollen. ${ }^{100}$ Vielmehr sieht es bei der Nutzung einer gemeinsamen Software ein großes Problem, wenn durch die softwarebedingten Vorgaben des einen Kooperationspartners der andere Verwaltungsträger Entscheidungsspielräume verliert, die ihm im Rahmen eigenverantwortlicher Aufgabenerfüllung zustünden. ${ }^{101}$

Daraus darf aber nicht vorschnell auf die Verfassungswidrigkeit des Netzwerkes, des Behördennetzes, des E-Government oder nur einer arbeitsteiligen Verwaltung geschlossen werden, da eine solche Sichtweise die überkommene hierarchische Verwaltungsorganisation mit ihrem System sachlicher und örtlicher Zuständigkeiten für verfassungsrechtlich unantastbar erklären würde. Gleiches gilt für eine bestimmte monistische Konzeption demokratischer Legitimation, die eine plausible und derzeit herrschende Theorieauffassung wiedergibt, dem Grundgesetz aber nicht unmittelbar zu entnehmen ist. Die Konkretisierung des Rechtsstaats- und Demokratieprinzips und auch der Kompetenzordnung können und müssen vielmehr einfachgesetzlich erfolgen. Der Gesetzgeber kann damit Veränderungen an den Legitimationsmodi sowie der konkreten Verfahrens- und Organisationsgestalt vornehmen. Aus den bisherigen Erörterungen resultiert also ein rechtlicher (und gesetzgeberischer) Handlungsbedarf, der allerdings intensiver rechtswissenschaftlicher Diskussion bedarf.

Misst man dieses Behördennetz zur Informationsverarbeitung und Wissensgenerierung an dem Modell des linearen staatlichen Entscheidungsprozesses, so werden normative Defizite deutlich. Das System der föderalen Informationsverarbeitung und Wissensgenerierung funktioniert nicht optimal, Defizite beim Informations- und Wissensstand von Behörden und Parlamenten lassen sich in zahlreichen Fällen nicht leugnen, da ein Umgang mit der heutigen Komplexität von Informationen und Wissen in den überkommenen Strukturen nur schwer möglich ist. All dies führt letztlich auch zur Unzufriedenheit bei den Bürgerinnen und Bürgern, die einer derartigen Vielzahl von Informations- und Wissensträgern ebenfalls oftmals ratlos gegenüberstehen. Nicht ohne Grund reagiert das Europäische Gemeinschaftsrecht auf diese Hilflosigkeit und Undurchschaubarkeit staatlichen Wissens mit klaren Vorgaben bis hin zur Verwaltungsorganisation in Gestalt einheitlicher, zur umfassenden Information und Wissensweitergabe ver-

100 BVerfGE 119, 331 (369); zu den Aussagen des Urteils mit IT-Bezug auch im Kontext eines „EGovernment-Gesetzes“ für Schleswig-Holstein, Schulz, S.E.: Ein eGovernment-Gesetz für SchleswigHolstein, a. a. O., $275 \mathrm{f}$.

101 BVerfG, 119, 331 (374). 
pflichteter Stellen, wie etwa auf der Grundlage von Art. 6 EU-DLR. ${ }^{102}$ Das tatsächliche Netzwerk, das Informationen verarbeitet, Wissen generiert und Staatsgewalt erzeugt, entspricht immer weniger den grundgesetzlichen Vorgaben. Zudem ist die hohe Komplexität von Informationen und Wissen politisch nicht mehr steuerbar, sodass auch Defizite demokratischer Legitimation bei der Ausübung von Staatsgewalt möglich sind.

Vor allem der vom Bundesverfassungsgericht kreierte Grundsatz der vollständig eigenständigen Aufgabenerledigung widerspricht prima facie § 71c VwVfG, der Informations- und Wissensübermittlung bei der einheitlichen Stelle bündelt. ${ }^{103}$ Und selbst wenn man im Sinne der Glykolwein-Entscheidung für staatliche Informationstätigkeit diese Grenzen nicht unbedingt so eng ziehen will, bleibt die Grenzziehung zwischen Informationsverarbeitung und Wissensgenerierung, bei denen zudem immer mehr auf Private zurückgegriffen werden muss, zur Ausübung von Staatsgewalt unklar. Schließlich legt die aus gemeinschaftsrechtlicher, aber auch informationsrechtlicher Perspektive nahe liegende Einheitlichkeit von Informationen und Wissen aus Sicht des Bürgers organisationsrechtliche und dann letztlich auch legitimatorische Konsequenzen nahe. So wie die Ergebnisund Auswirkungsorientierung im Schutzbereich des Bürgers aus der Grundrechtsdogmatik bekannt ist, so ist diese Sichtweise dem Verwaltungsorganisationsrecht, mit Ausnahme einzelner gemeinschaftsrechtlicher Verpflichtungen, und dem Staatsorganisationsrecht noch immer unbekannt.

\section{Reformbedarf}

Nach allem dürfte deutlich geworden sein, dass Informationsverarbeitung und Wissensgenerierung in Zeiten zahlreicher gemeinschaftsrechtlicher Vorgaben und moderner Informations- und Kommunikationstechnik entweder auf ein verfassungsrechtliches Niemandsland stoßen oder aber mit einem unterkomplexen linearen staatlichen Entscheidungsprozess in Einklang gebracht werden müssen.

102 Umfassend zur Umsetzung in Deutschland Schliesky, U./Schulz, S.E./Neidert, A.: Bewertung der Richtlinienumsetzung in Deutschland: Der Einheitliche Ansprechpartner im Verwaltungs- und Organisationsrecht, in: Schliesky, U. (Hg.): Die Umsetzung der EU-Dienstleistungsrichtlinie in der deutschen Verwaltung - Teil III: Wissen, Information, Verantwortung, Kiel, 2010, 249-307.

103 Zur „,vollständigen Auskunftsfähigkeit“ als Zielsetzung staatlichen Wissensmanagements Schulz, S.E., Vernetztes Wissen, a. a. O. 


\section{Verfassungsrechtliche Absicherung eines „föderalen Informations- managements"}

Informationen und Wissen waren und sind unabdingbare Voraussetzungen für problemadäquate staatliche Entscheidungen. Angesichts der immens gewachsenen Komplexität der Informationsverarbeitung müssen jedoch auch der Staat und seine Verwaltungen arbeitsteilig und kooperativ in Netzwerken die nötigen Informationen zu einem staatlichen Wissen generieren. Die Grenzen zwischen der Vorbereitung von Entscheidungen und der eigentlichen Entscheidung, vor allem die Verantwortung für die Inhalte der Entscheidung, werden angesichts der netzwerkartigen Wissensgenerierung fließend. Die grundgesetzlichen Verwaltungskompetenzen und die auf diesen basierende einfachgesetzliche Zuständigkeitsordnung können nur noch schwerlich im Sinne eines Trennprinzips aufrechterhalten werden. Vielmehr muss sowohl für die Verwaltungen selbst als auch für den Außenkontakt zu Bürgern und der Wirtschaft sichergestellt werden, dass trotz der Zuständigkeitszersplitterung die Voraussetzungen für ein gemeinsames Informationsangebot geschaffen werden können. ${ }^{104}$ Die Bemühungen um ein bundesweit einheitliches Open Government Data-Portal (www.govdata.de) oder die - letztlich nur ansatzweise umgesetzten - Anforderungen der Art. 6 und 7 EU-DLR sind praktische Beispiele dieser abstrakten Herausforderung. Dieses Informationsangebot ist oft nur, allerdings auch zwingende, Voraussetzung einer gemeinsamen Aufgabenerledigung. Und letztlich - aus Sicht einer bürgerfreundlichen Verwaltung - muss es das Ziel sein, dem Bürger und insbesondere auch der Wirtschaft komplette „Genehmigungspakete“ für bestimmte Lebenssituationen über elektronische einheitliche Anlaufstellen anzubieten. So werden die effizienzsteigernden Möglichkeiten der Informations- und Kommunikationstechnik erst dann genutzt, wenn tatsächlich die Perspektive des Bürgers eingenommen wird ${ }^{105}$ und aus dessen Sicht eine einheitliche Verwaltungsplattform existiert, hinter der durchaus unterschiedliche Behördenangebote liegen können, die aber interoperabel verzahnt sein müssen. Erst die Orientierung an derartigen Internet-Plattformen, die im E-Commerce heute die erfolgreichsten Geschäftsmodelle darstellen, wird dem E-Government zum Durchbruch verhelfen und im Übrigen auch bundesweit qualitativ gleichwertige Verwaltungsangebote unter den Vorzeichen des demographischen Wandels ermöglichen. Hierfür 
bedarf es zum Teil noch neuer organisatorischer Lösungen und neuer Verfahrensgestaltungen, vor allem aber einer normativen Anerkennung und Abbildung des vernetzten Behördenalltags. Letztlich stehen wir vor der gleichen Herausforderung wie bei Inkrafttreten des Grundgesetzes, als es galt, bestehende Behördenstrukturen und schriftliche Verwaltungsverfahren unter Geltung normativer demokratisch-rechtstaatlicher Anforderungen einfachgesetzlich abzubilden. Angesichts des neuen Mediums handelt es sich um eine Gestaltungsaufgabe nicht nur, aber auch für die Juristen.

Sodann bedarf es der Diskussion, inwieweit die Verfahren der Informationsverarbeitung in der „Informationsgesellschaft“ weiterhin als rechtlich untergeordneter Teil des staatlichen Entscheidungsprozesses verstanden werden sollen. Wenn Informationen und Wissen tatsächlich die neue „Währung“ der Informationsgesellschaft sind, wird man mittelfristig um einen perspektivisch veränderten Blick auf das Tätigwerden des Staates bezüglich Informationsverarbeitung und Wissensgenerierung kaum umhin kommen. Es wären dann nämlich sowohl grundrechtliche als auch staatsorganisationsrechtliche Neuerungen im Grundgesetz angezeigt.

Generell gilt, dass die politischen Einflussmöglichkeiten sowie die politischen Abstimmungs- und Entscheidungsverfahren den tatsächlichen Wegen der Informationsverarbeitung und Wissenssammlung besser entsprechen müssen. Angesprochen ist damit vor allem die staatliche Wissensgenerierung, die im demokratischen Rechtsstaat eine entscheidende Rolle spielt. Denn der Glaube an die Richtigkeit und Integrität staatlichen Wissens sowie der daraus abgeleiteten Entscheidungen muss aufrecht erhalten werden, wenn der Legitimitätsglaube bezüglich der ausgeübten Herrschaftsgewalt nicht verlorengehen soll. Angesichts der im Informationszeitalter bislang erschreckend begrenzten Ressourcen des Staates wird man aber auch um eine Neuabgrenzung zwischen privatem und staatlichem Wissen nicht umhin kommen. Denn es darf nicht übersehen werden, dass Private, vor allem bestimmte Interessengruppen, mithilfe des Internets auf einfache Weise intensiven Einfluss auf politische Diskussionsprozesse nehmen und Wissen präsentieren können. ${ }^{106}$ Die staatliche Informationstätigkeit und Wissensweitergabe auch künftig nur auf den ominösen Kompetenztitel „Staatsleitung der Regierung" - ohne klare Vorgaben und Abgrenzung zu Grundrechten - stützen zu wollen, wird auf Dauer zu wenig sein. 


\section{Normative Abbildung realer und digitaler Arbeitsteilung}

Denkt man die hier angeschnittenen Probleme weiter, so wird deutlich, dass die Arbeitsteilung zwischen staatlichen Verwaltungen - vor allem im Bereich der IT, aber auch bei ganz klassischen ,realen“ Verwaltungshandlungen - erheblich zugenommen hat. Sowohl die Verfassung als auch die Rechtsordnung insgesamt sind aufgrund überkommener Souveränitätsvorstellungen darauf nach wie vor nicht hinreichend vorbereitet. Als Reaktion auf eine aktuelle Problemlage hat der Verfassungsgeber mit Art. 91e GG eine solche normative Erlaubnis für einen besonderen Verwaltungszweig in die Verfassung aufgenommen, doch harrt dieses Problem noch in vielen anderen Bereichen des täglichen staatlichen Lebens einer Lösung. Entsprechende Ansätze wurden bereits an anderer Stelle ausgebreitet. $^{107}$

\section{Verbesserung der föderalen IT-Steuerung}

Ein wesentlicher Grund für die Aufnahme des Art. 91c GG in die Verfassung war - wie ausgeführt - die Verbesserung der föderalen IT-Steuerung. Diesbezüglich gab es in den vergangenen vier Jahren deutliche Fortschritte, doch besteht nach wie vor Optimierungsbedarf, und zwar gerade auch in rechtlicher Hinsicht. Eine isolierte IT-Steuerung ist bereits in den jeweiligen Verwaltungsräumen isoliert schwierig. Bund, Länder und Kommunen können sich jeweils nur mühsam oder oft gar nicht auf einheitliche IT-Strategien verständigen. Angesichts der zahlreichen Akteure, die zumindest in der Praxis mit einer Veto-Macht ausgestattet sind, sind konsistente IT-Steuerungskonzepte nur schwer realisierbar. Dieses Problem vervielfacht sich im föderal-gesamtstaatlichen Kontext. Sowohl die Verwaltungskompetenzen des Grundgesetzes als auch die Ressorthoheit von Ministerinnen und Minister stellen oftmals unüberwindliche Grenzen für eine ganzheitliche IT-Steuerung dar. Dieser Befund kontrastiert die einfache Erkenntnis, dass Informations- und Kommunikationstechnik an Grenzen nicht Halt macht. Dies gilt für Staaten-, Landes- und Ressort- oder Kompetenzgrenzen gleichermaßen. Der Vorteil IuK-gestützter Verwaltungsprozesse kann erst bei einer einheitlichen oder zumindest interoperablen IT-Struktur und darauf laufenden standardisierten Verwaltungsprozessen genutzt werden. Die IT-Steuerung ist damit letztlich keine technische Frage, sondern betrifft vor allem die Aufbauund Ablauforganisation der Verwaltungen. Eine konsistente IT-Steuerung stei- 
gert die Wirtschaftlichkeit, dient einer größeren Bürger- und Unternehmensfreundlichkeit der Verwaltungen, fördert staatliche Innovationen, ${ }^{108}$ verbessert die Kooperation der Verwaltungen und stellt letztlich die staatliche Handlungsfähigkeit im Kontext der Informationsgesellschaft sicher. Zu einer derartigen ITSteuerung gehört dann auch, Veränderungsprozesse verbindlich anzustoßen und zu einem Erfolg zu führen. Vorbilder aus dem öffentlichen Sektor sind insoweit die öffentlich-rechtlichen Sparkassen und die Industrie- und Handelskammern, die bei Wahrung ihrer Eigenständigkeit nicht nur eine gemeinsame IT-Strategie verabredet haben, sondern sich auch nur noch eines einheitlichen ITDienstleisters und standardisierter Prozesse bedienen.

Zum einen wird am Beispiel der IT-Steuerung deutlich, dass die Räume von Politik und Verwaltung nicht mehr deckungsgleich sein müssen - und zur erfolgreichen Nutzung von Informations- und Kommunikationstechnik auch häufig nicht mehr deckungsgleich sein dürfen. Niemand wird ernsthaft vorschlagen, politische Räume nach den Möglichkeiten der Informations- und Kommunikationstechnik neu zu fassen. Schon längst ist es aber möglich und durchaus auch in der Praxis erprobt, politische Verantwortungseinheiten aufrecht zu halten, die sich einer gemeinsam gesteuerten Verwaltung bedienen. Der damit verbundene „Bruch“ des monistischen Legitimationsstrangs zwischen politischer Vertretungskörperschaft und konkret ausführender Verwaltungseinheit muss dann allerdings rechtlich erlaubt sein und zumindest über demokratische Kontrollmöglichkeiten kompensiert werden. Aufgrund der Instrumentalfunktion der Informations- und Kommunikationstechnik betrifft dieses Problem zahlreiche, potentiell sogar alle Sachmaterien staatlicher Verwaltung und alle Verwaltungseinheiten. Damit verbirgt sich hinter der so fachlich anmutenden IT-Steuerungsproblematik ein grundlegendes Thema für eine Föderalismusreform III und schließlich auch der Schlüssel zu einer umfassenden Modernisierung der staatlichen Verwaltung: Die Frage lautet, ob es gelingt, die souveränitäts- und legitimationstheoretisch entstandene Idee einer Deckungsgleichheit von politischem Raum und Verwaltungseinheit zu durchbrechen und sowohl mit dem Rechtsstaats- als auch mit dem Demokratieprinzip des Grundgesetzes (Art. 20 GG) zu versöhnen. Mit den überkommenen Denkmodellen - auch des Bundesverfassungsgerichts - erscheint dies wegen der verkomplizierten Verantwortungszurechnung, wie sie im Rechtsstaats- und Demokratieprinzip wurzelt, schwierig. Das Beispiel des Art. 91e Abs. 
1 GG zeigt jedoch, dass eine derartige Verantwortungsverzahnung möglich ist, auch wenn sie der verfassungsrechtlichen Erlaubnis bedarf.

Zum anderen entsteht verfassungsrechtlicher Reformbedarf im Hinblick auf die IT-Steuerung aufgrund eines Missverständnisses des Ressortprinzips als Exklusionsinstrument. Die Ministerverantwortung i.S.d. Art. 65 S. 2 GG umfasst nicht zwingend jedes Detail der IT-Ausstattung, wird in der Praxis aber oftmals in diese Richtung überdehnt. An sich wäre auch eine andere Auslegung möglich, zumal das Kollegial- bzw. Kabinettsprinzip entsprechende verfassungsrechtliche Gestaltungsspielräume durchaus eröffnet. In der Praxis kommt diese Theorie allerdings in der Regel nicht an, sodass auch für die Vereinheitlichung der ITSteuerung ohne die Veto-Macht des Ressortprinzips eine Ergänzung des Art. 91c GG oder - dann allerdings auch mit Blick auf europarechtliche oder generelle Fragen - sogar des Art. 65 GG denkbar und wünschenswert erscheint.

\section{Fazit}

Die Aufnahme der IT in das Grundgesetz durch die Föderalismusreform II im Jahre 2009 war dringend erforderlich. Art. 91c GG hat sich als Grundlage einer verbesserten IT-Steuerung und diesbezüglichen Abstimmung zwischen Bund und Ländern bewährt. Aufgrund des seinerzeitigen Kompromisscharakters besitzt die Vorschrift allerdings nur einen begrenzten Anwendungsbereich. Als Grundlage für die neu zu gestaltenden Prozesse der Arbeitsteilung von Verwaltungen mithilfe von Informations- und Kommunikationstechnik oder gar für ein umfassendes staatliches Informations- und Wissensmanagement genügt die Bestimmung nicht. Insoweit besteht ein Reform- oder besser Erweiterungsbedarf, der in diesem Beitrag begründet wurde.

Unabhängig von der durch Art. 91c GG geregelten IT-Zusammenarbeit von Bund und Ländern stellen sich im Zusammenhang der Digitalisierung der Gesellschaft sehr grundsätzliche Fragen, die hier nicht behandelt werden können, aber ebenfalls im Zuge einer Föderalismusreform III beratungswürdig wären: Zum einen geht es um den (grundrechtlichen) Schutz der Privatheit in der digitalen Gesellschaft, und zwar sowohl gegenüber dem Staat als auch und gerade gegenüber monopolartigen Internet-Dienstleistern. ${ }^{109}$ Zudem werden die repräsentative Demokratie und damit die Verfassung in absehbarer Zeit auf ein Schwinden der klassischen demokratischen Öffentlichkeit reagieren müssen, 
wenn eine so wesentliche Grundbedingung des demokratischen Bauplans für unser Gemeinwesen wie die Öffentlichkeit sich weiterhin so radikal verändert. Diese zwei Hinweise dokumentieren bereits deutlich, dass längst nicht alle Herausforderungen der Informations- und Kommunikationstechnik mithilfe von Art. 91c GG bewältigt werden können, sondern der grundlegenden wissenschaftlichen und politischen Diskussion bedürfen, um dann Eingang in die Verfassung zu finden. 